\title{
A Dynamic Limit Order Market with Diversity in Trading Horizons*
}

\author{
Mark Van Achter ${ }^{\dagger}$
}

June, 2008

\begin{abstract}
This paper considers a trading game in which sequentially arriving liquidity traders either opt for a market order or for a limit order. One class of traders is considered to have an extended trading horizon, implying their impatience is linked to their trading orientation. More specifically, sellers are considered to have a trading horizon of two periods, whereas buyers only have a single-period trading scope (the extended buyer-horizon case is completely symmetric). Clearly, as the life span of their submitted limit orders is longer, this setting implies sellers are granted a natural advantage in supplying liquidity. This benefit is hampered, however, by the direct competition arising between consecutively arriving sellers. Closed-form characterizations for the order submission strategies are obtained when solving for the equilibrium of this dynamic game. These allow to examine how these forces affect traders' order placement decisions. Further, the analysis yields insight into the dynamic process of price formation and into the market clearing process of a non-intermediated, order driven market.
\end{abstract}

JEL Codes: G10, G20

Keywords: Limit Order Market, Order Placement Strategy, Order Flow, Market Microstructure.

${ }^{*}$ I would like to thank Hans Degryse, Thierry Foucault, Carole Gresse, Sophie Moinas, Anna Scherbina, Bob Schwartz, Erik Theissen, Christian Voigt, Huiping Zhang, participants at the 2007 Degroote Conference on Market Structure and Integrity (Toronto), the 2007 AFFI meeting (Paris), the 2008 Conference of the Swiss Society for Financial Market Research (Zürich), the 2008 Dauphine Workshop on Financial Market Quality (Paris), the 2008 Conference on the Industrial Organization of Securities Markets (Frankfurt), the 2008 EFMA Meeting (Athens), as well as seminar participants at the university of Bonn for helpful comments and suggestions.

${ }^{\dagger}$ University of Bonn, Institute of Business Administration I (BWL I), Adenauerallee 24-42, 53113 Bonn, Germany (e-mail: Mark.VanAchter@uni-bonn.de). 


\section{Introduction}

Nowadays, continuous limit order markets are becoming increasingly important in fulfilling investors' trading needs (see e.g. Jain (2003)). A valuable feature driving this success is that these markets do not require synchronization of the timing of these trading needs across traders. Indeed, investors willing to supply immediacy over time are able to trade with those demanding it without any form of intermediation. In return, the former type of investors receive the bid-ask spread as a compensation. In practice, we often observe a divergence in liquidity needs arises between buyers and sellers. For instance, a liquidity crisis on financial markets (as occurred in August 2007 ) causes prices to decrease. In turn, these lowering prices put pressure on institutional investors' open externally-financed positions. If margin requirements are eventually hit, standing loans are no longer fully collateralized and consequently (partially) called by creditors. This forces the institutional investors to engage in immediate (partial) liquidation of the portfolio in an already falling market, which puts additional downwards pressure on prices and potentially launches a destructive endogenous liquidity spiral (see e.g. Brunnermeier and Pedersen (2006), Kang, Hameed and Viswanathan (2006), and Ewerhart and Valla (2007) for further background). Clearly, within this setting, sellers' liquidity needs are more urgent than buyers'. While the former type of traders are obliged to sell immediately, the latter may anticipate lowering prices and even postpone their trades, which implicitly lengthens their trading horizon. In contrast, the creation of the speculative bubble preceding this liquidity crisis corresponds to the reverse setting in which sellers have a relatively longer trading horizon than buyers. ${ }^{2}$

To analyze how a limit order market performs under these conditions, within this paper, I develop a dynamic model of limit order trading which captures this potential divergence in liquidity needs between buyers and sellers. Sellers are considered to have an extended trading horizon of two periods vis-à-vis buyers that only have a single-period trading scope. ${ }^{3}$ This setting grants sellers a natural advantage in supplying immediacy. However, it also induces direct competition between consecutively arriving sellers. The model will allow us to examine how these forces affect traders' order placement decisions. Solving for the equilibrium of this dynamic game, closed-form solutions for the order placement strategies are obtained. Further, the analysis yields insight into the

\footnotetext{
${ }^{1}$ In this month, the financial sector undertook a dramatic re-appraisal of the risk contained in (complex forms of) structured credit. More specifically, creditors became concerned about market valuations of illiquid assets such as collateralized debt and loan obligations (CDOs and CLOs), which lead to a downward correction and a liquidity dry-up. Resultingly, several hedge funds and special investment vehicles stumbled into severe problems as their financing could no longer be prolonged. They were forced to quickly unwind their positions, causing contagion effects to other financial markets.

${ }^{2}$ Other examples of speculative bubbles on financial markets include the Dot-com bubble (circa 1995-2001) and the Chinese stock bubble (as of 2006). See e.g. Scheinkman and Xiong (2003), Shiller (2005) or Hong, Scheinkman and Xiong (2006) for further theoretical background on the creation of a speculative bubble.

${ }^{3}$ This setup corresponds to the speculative bubble case. However, as the model is completely symmetric, the results for the reverse liquidity crisis case are easily obtained.
} 
dynamic process of price formation and into the market clearing process of a non-intermediated, order driven market. It features sequentially arriving liquidity traders ${ }^{4}$ willing to trade one share of the asset. Trading merely occurs because investors differ in their share valuations. Traders demanding immediacy typically submit market orders, while those supplying it use limit orders. Market orders guarantee immediate execution at the best limit order price available in the book upon arrival. Limit orders allow traders to improve upon this execution price, but do not offer immediate or certain execution. ${ }^{5}$ The choice between both is made conditional on the state of the book and aims at maximizing the total payoff from trading. The bid-ask spread is the mere result of strategic rentseeking by limit order traders. Its size and position is shown to be a function of the differences in valuation among investors. Moreover, the spread's magnitude is argued to hinge on the competition between sequentially arriving limit order sellers.

The current paper is situated within the theoretical literature developing models to describe limit order markets. Within this literature, two strands of research could be distinguished.

A first line of work develops models that are static. Examples include Glosten (1994), Bernhardt and Hughson (1997), Chakravarty and Holden (1995), Rock (1996), Seppi (1997), Biais, Martimort and Rochet (2000), and Parlour and Seppi (2003). These models allow to analyze the optimal bidding strategies for limit order traders and to determine the bid-ask spread level. However, on the downside, all traders are forced to arrive simultaneously implying their trading horizons are strictly limited to a single period and the order choice is exogenously imposed in these models. In this model, I will relax these restrictions by considering sequentially arriving traders with (partially) extended trading horizons that endogenously choose between market and limit orders.

A second line of work develops dynamic microstructure models for a limit order market with endogenous order flow. ${ }^{6}$ Parlour (1998) describes traders' order placement strategies at the inside quotes. She analyzes the influence of available depth at these quotes on traders' decisions. Goettler, Parlour and Rajan (2005) develop a limit order market model in which they consider trading as a stochastic sequential game. They develop a technique to solve for the equilibrium numerically and show order flow persistence arises even in the absence of changes in the consensus value of the asset. To my knowledge, only two papers allow for traders of varying impatience within this line of work. First, Foucault, Kadan and Kandel (2005) consider strategic liquidity traders of varying impatience to analyze order placement strategies, spread dynamics and market resiliency. In a closely related model, Rosu (2007) examines endogenous undercutting and

\footnotetext{
${ }^{4}$ Madhavan (2000) defines liquidity traders as agents smoothing their intertemporal consumption stream through portfolio adjustments.

${ }^{5}$ Note that a buy (sell) limit order specifies the maximum (minimum) price the buyer (seller) wants to pay (receive), whereas a market order will buy or sell at any price.

${ }^{6}$ In contrast, there also exist dynamic limit order market models with an exogenous order flow such as Angel (1994), Domowitz and Wang (1994), and Harris (1998).
} 
strategic cancellation of limit orders. Within both these models, variations in waiting costs (i.e., the costs related to delaying execution) among traders are used to proxy their impatience. The (expected) time to execution is shown to influence their bidding strategies through these waiting costs. In contrast, in my model waiting costs are assumed non-existent, a trader's impatience is merely represented by her trading horizon. The time to potential execution is restricted to zero or one periods for short-horizon traders ${ }^{7}$ and to zero, one or two periods for the long-horizon traders on the other side of the market. This divergence in trading horizon will also influence their quoting strategies as will be argued below. The main benefit of this approach is that it does not need to rely on several simplifying assumptions as do Foucault, Kadan and Kandel (2005) and Rosu (2007). Finally, the dynamic models that are positioned closest to the current one by their design are Foucault $(1995,1999)$, and Handa, Schwartz and Tiwari (2003). ${ }^{8}$ Foucault (1995) develops a model to examine price formation and optimal trading strategies with two types of buyers and two types of sellers. In comparison, Foucault (1999), and Handa, Schwartz and Tiwari (2003) only examine a single trader type on each market side. However, they add volatility in the asset price and private information to this setting, respectively. ${ }^{9}$ Note that to simplify the analysis of competitive interactions between the traders, all three models consider sequentially arriving traders that only have a single-period trading horizon. Within this setting, indirect competition over time arises as arriving traders could opt to trade with the previous trader (via a MO) or with the subsequent trader (via a LO). The model developed in this paper (partially) relaxes this assumption by incorporating that one of both trader types now exhibits an extended trading scope. This setup alters the previously documented indirect competition pattern, as limit order traders on one side of the market are now able to address traders arriving in the two subsequent periods, hence increasing their order's exposure. Moreover, it also induces direct competition between those traders having an extended trading horizon. For instance, should sellers have a two-period scope, it is possible to have two LO sells in the book at the same time that are directly competing for the arriving MO buy. Depending on the market conditions the sellers will act as Bertrand competitors (see e.g. Bertrand (1883) and Maskin and Tirole (1988)) and engage in undercutting behavior that is not exogenously imposed as in Foucault, Kadan and Kandel (2005). Moreover, traders may opt to join the existing queue at the inside quotes or choose to revert to a backlying price, a feature which is also not present in Foucault, Kadan and Kandel (2005). As such, the model allows us to examine how quotes evolve in between transactions.

\footnotetext{
${ }^{7}$ It equals "zero" if the trader submits a market order, "one" if she submits a limit order.

${ }^{8}$ Note that Handa, Schwartz and Tiwari (2003) extends Handa and Schwartz (1996) who analyze the rationale and profitability of limit order trading. However, the latter paper does not explicitly model a trader's decision as to whether to place a limit or a market order.

${ }^{9}$ Another difference between the three models lies in the way the proportion of buyers and sellers in the market is modelled. While it is free to vary without restriction in Foucault (1995), and Handa, Schwartz and Tiwari (2003), there can be no imbalance between supply and demand in Foucault (1999).
} 
Further, this paper relates to two other strands in the theoretical market microstructure literature.

First, it complements the literature focusing on the cost of providing liquidity (or the "price of immediacy"). Duffie, Gârleanu and Pedersen (2005) develop a model in which asynchronously arriving investors must "search" for counterparties. The introduction of market makers is a natural way to deal with this issue. However, in exchange for providing immediacy, the imperfectly competing market makers in their model employ their (temporary) market power to extract part of the investors' trading gains, which induces the creation of a bidask spread. In a related model, Chacko, Jurek and Stafford (2007) analyze how a monopolistic market maker is able to extract trading gains from impatient investors demanding immediacy (i.e., limit order traders in their model). The current paper complements this literature by analyzing liquidity provision on a limit order market. This type of market requires no intermediary and allows both patient and impatient investors to supply and demand liquidity (by submitting limit orders and market orders, respectively). Within a stylized setting, our model allows to investigate whether patient LO traders, when supplying liquidity, are able to extract more trading gains from the counterparty than impatient ones. Intuitively, the fact that they are able to expose their LO over several periods (inducing relative market power) seems to confirm this hypothesis. Direct competition with other patient liquidity-supplying traders on the same market side, however, may push prices to a more competitive level and thus reverse this result.

Second, this paper contributes to the literature on dynamic competition between liquidity suppliers. Cordella and Foucault (1999) focus on direct price competition between dealers with a sequential bidding process to characterize the evolution of the best quotes in between transactions. They argue the speed of convergence to the competitive prices hinges on the frequency with which dealers check their offers and on the tick size. While they focus on two dealers bidding sequentially on the sell side of the market, this paper highlights dynamic quoting behavior by sequentially arriving liquidity suppliers on both sides of a limit order market with direct competition arising on one market side.

Within our model, two stationary equilibria are derived for the delineated trading process. All depends on how a first-in-line LO seller deals with future direct competition from consecutively arriving sellers. In a first equilibrium, she opts to quote a high quote $A_{I}$. This initial ask quote will certainly be undercut should the next trader arriving also be a seller. The consecutive arrival of a series of sellers will induce an undercutting process. Two possible equilibrium paths for the ask quote are highlighted: one with and one without reversion to the initial ask quote. The LO buyer, which does not need to account for direct competition by other buyers as her trading horizon only lasts one period, is shown to optimally quote bid $B_{I}$ within this equilibrium. In a second equilibrium, a first-in-line LO seller chooses to protect herself from this undercutting process by posting a low quote $A_{I I}$ that is "undercutting-proof". As such, she is ensured of a two-period order exposure at the cost of a lower execution price. In 
this equilibrium, a LO buyer optimally quotes $B_{I I}$. Note that both $B_{I}$ and $B_{I I}$ are set as the lowest bid quote at which an incoming seller is willing to submit a MO within the respective equilibrium (which in turn depends on the sellers' own quoting strategy). Hence, both buyers and sellers account for each other's actions in determining their optimal quote.

The quote choice is shown to depend on the total trading gains level (i.e., the difference between buyers' and sellers' private valuations). The higher the potential gains from trading, the more sellers are inclined to quote $A_{I}$ despite its lower corresponding execution probability, as the opportunity cost of sticking to the low undercutting-proof quote $A_{I I}$ becomes too high. This effect becomes less pronounced when sellers outnumber buyers by far in the market, implying higher direct and indirect competition between sellers as a result of the rationing problem arising. In this case the difference between $A_{I I}$ and $A_{I}$ (which represents the loss from switching from $A_{I}$ to $A_{I I}$ ) is shown to be relatively small. Moreover, the execution risk increase from switching from $A_{I}$ to $A_{I I}$ is demonstrated to be substantial. Consequently, LO sellers will be eager to expose their order for two periods and hence quote the undercutting-proof quote $A_{I I}$, even for relatively elevated total trading gain levels.

This paper proceeds as follows. Section 2 presents the setup of the model. Section 3 provides a formal definition of the equilibrium which is further analyzed in Section 4. Finally, Section 5 concludes. All proofs are relegated to the Appendix.

\section{Setup}

An infinite horizon model is developed in order to examine a continuous limit order market listing a single security. Each period $t=0,1, \ldots+\infty$, exactly one agent arrives that observes the limit order book (LOB) and decides to trade one share of the asset. These agents, or traders as they will be referred to from now on, are risk neutral and expected utility maximizers. Whether they want to buy or sell depends on their exogenously determined trading orientation: with probability $k$ a trader is a buyer having a private valuation of the asset equal to $V_{h}$, with probability $1-k$ she is a seller having a private valuation $V_{l} \cdot{ }^{10} \mathrm{~A}$ buyer's utility of trading the asset at price $P$ is $U\left(V_{h}, P\right)=\left(V_{h}-P\right)$, while a seller's utility is $U\left(V_{l}, P\right)=\left(P-V_{l}\right)$. Hence, as non-trading gains are normalized to zero, $V_{h}$ and $V_{l}$ reflect the reservation price that buyers are willing to pay and that sellers are willing to receive for one share of the asset, respectively. Both valuations are non-negative and $V_{h}>V_{l}$, which implies there are always gains from trade between both parties. ${ }^{11}$ Moreover, traders are assumed to differ in trading horizon: whereas buyers only have a scope of a single period, sellers will consider the two subsequent periods. Hence, within this model, sellers are

\footnotetext{
${ }^{10}$ As such, $k \in[0,1]$ is a measure of the imbalance between supply and demand.

${ }^{11}$ The differences in valuation are an outcome of taxes, liquidity shocks, or other portfolio considerations such as differences in endowment, opinion or the expected values of the asset.
} 
more patient than buyers. ${ }^{12}$ Clearly, the case where sellers only have a limited trading horizon, and buyers an extended one is completely symmetric. Below, I will examine how this trading horizon diversity will be reflected in their trading behavior.

Traders have two options at their disposal to trade. On the one hand, they could post quotes and submit a limit order ( $\mathrm{LO}$ ) which does not offer certainty of execution. On the other hand, they could submit a market order (MO) which guarantees immediate execution but at the cost of a less favorable execution price. The optimal order choice ultimately involves a trade-off between the cost of delayed execution and the cost of immediacy. As liquidity-demanding MOs execute against standing liquidity-supplying LOs, they can only be submitted if a counterparty LO is already present in the LOB. Hence, upon arrival, traders typically first observe the state of the LOB. Given the availability of an ask (bid) price, a buyer (seller) could either submit a MO that executes against the best available quote or post a LO. In case no counterparty quote is available, posting a LO is the only option. The trading horizon diversity allows sellers to submit LOs that last for two periods, while those of buyers only hold a single period. Clearly, besides the self-selected price, this difference also affects the LO's execution probability which, as will be made clear below, is endogenous in the model as it depends on other traders' order placement strategies. When she has performed a transaction, a trader is no longer allowed to stay in the market and submitted orders cannot be cancelled or modified. Posted LOs in the LOB are executed in sequence according to time and price priority. The asset's tick size is assumed to be equal to $\Delta>0$. All parameters of the model, including $V_{h}, V_{l}, \Delta$ and $k$ are known to the investors. Moreover, they are constant over time, hence the market is assumed to be in steady state.

This trading structure is depicted in Figure 1 for a random point in time $t$ within this sequential trading process. An arriving trader could potentially face five different LOB states: (i) empty, (ii) 1 buy LO, (iii) 1 sell LO submitted two periods ago (i.e., an "old" LO sell), (iv) 1 sell LO submitted in the previous period (i.e., a "new" LO sell), and (v) 2 sell LOs. She will choose her trading strategy accordingly.

\section{Equilibrium}

The aim of this section is to provide a formal definition of the equilibrium. First, traders' order placement strategies are characterized. Such a strategy assigns an optimal order type and quote to each possible state of the LOB. To further specify these strategies, in a next step, the execution probabilities corresponding to each possible quote are derived. Finally, a stationary market equilibrium is defined as a set of mutual strategies such that each trader's strategy is optimal given the strategies of other traders.

\footnotetext{
${ }^{12}$ This case could also be seen as follows: whereas sellers have a common utility discount factor $\delta=1$ for the two upcoming periods, buyers have $\delta=1$ for the subsequent period and $\delta=0$ from then onwards.
} 


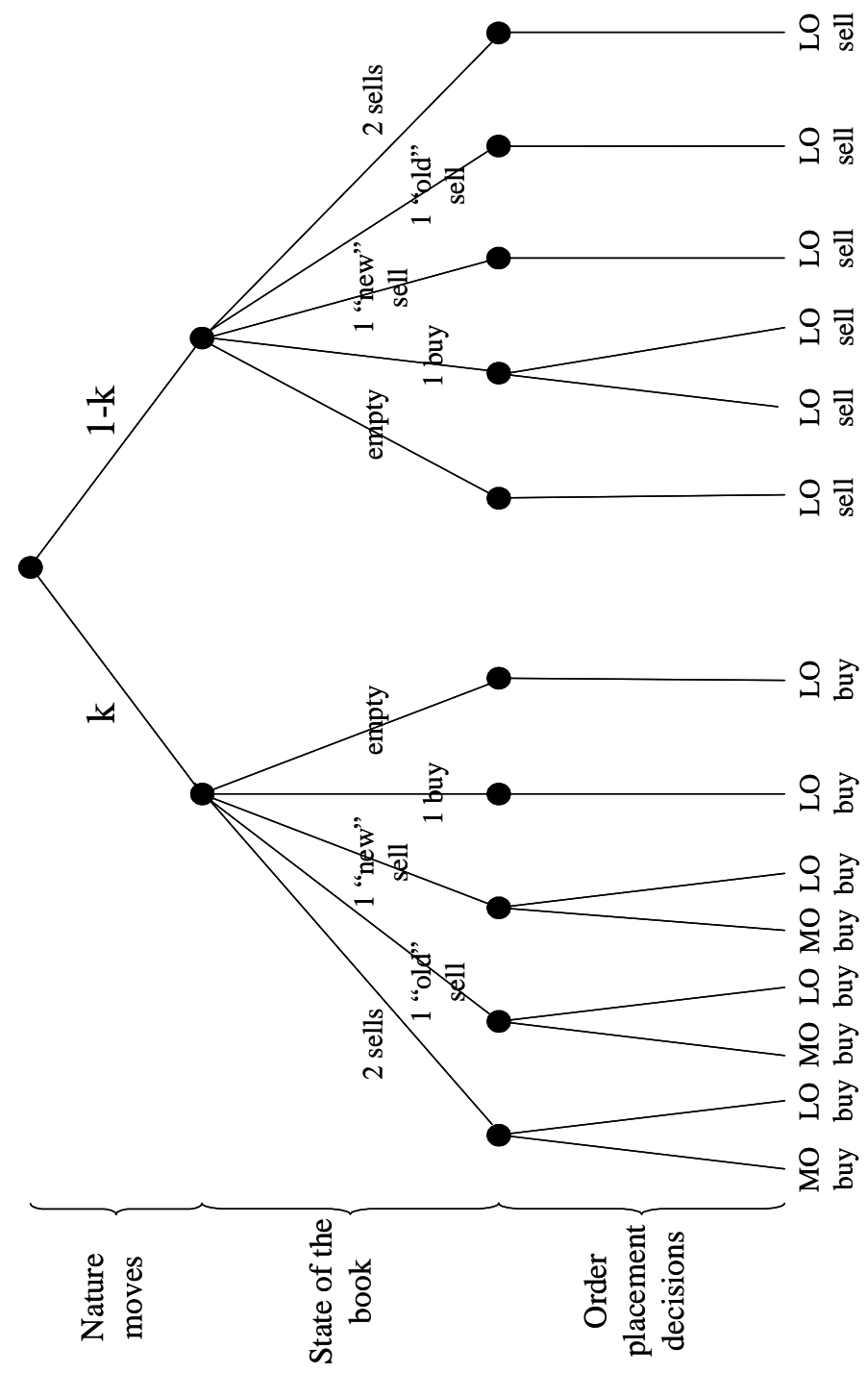

Figure 1: Tree diagram of the trading structure: events and decisions at time $\mathbf{t}$ 


\subsection{Order placement strategies}

Upon arrival, traders observe the limit order book and decide what to do. If it is empty or does not contain a counterparty LO, they opt for delayed and uncertain execution using a LO. If it does contain a counterparty LO, they could also opt for immediate and certain execution via a MO. In this subsection, the order placement strategy underlying this choice will be determined. An order placement strategy is a mapping, $o($.$) , that assigns an optimal order and quote$ decision to every possible starting position. As a first step, the cutoff decision rule traders use to submit a $\mathrm{MO}$ is described in the following proposition:

Proposition 1 Each arriving trader determines a cutoff price:

- a buyer arriving at time $t$ decides to trade immediately at the best ask if it is less than or equal to her cutoff price, $B_{0}(t)$, otherwise she will quote a bid $B(t)$;

- a seller arriving at time $t$ decides to trade immediately at the best bid if it is more than or equal to her cutoff price, $A_{0}(t)$, otherwise she will quote an ask $A(t)$.

Proof. See Appendix.

At these cutoff prices, the trader is indifferent between the value of a market order strategy and the expected value of a limit order strategy. Clearly, these prices will implicitly depend on the LO quote the trader would potentially submit (see proof of Proposition 1). Combined, this quote and the corresponding cutoff price fully characterize the traders' order placement strategies: i.e., $\Sigma(t)=\left(B_{0}(t), B(t)\right)$ for a time $t$ buyer and $\Phi(t)=\left(A_{0}(t), A(t)\right)$ for a time $t$ seller.

From now on, attention will be restricted to stationary strategies. ${ }^{13}$ This implies the time of arrival of the trader no longer influences the strategy: two traders who differ only by their arrival time face exactly the same problem, it is therefore natural to assume that they will use the same strategy. Consequently, the focus lies on the stationary equilibria of the trading game analyzed in this paper.

Definition 1 A stationary strategy:

- for a buyer is a function $\Sigma($.$) such that \Sigma(t)=\Sigma\left(t^{\prime}\right) \forall t \forall t^{\prime} \in\{1,2 \ldots,+\infty\}$;

- for a seller is a function $\Phi($.$) such that \Phi(t)=\Phi\left(t^{\prime}\right) \forall t \forall t^{\prime} \in\{1,2 \ldots,+\infty\} .{ }^{14}$

\footnotetext{
${ }^{13}$ The choice for stationary strategies is rationalized by the fact that all exogenous parameters (the probability $k$ and the agents' private valuations $V_{h}$ and $V_{l}$ ) are assumed to be stationary. Moreover, the horizon of the model is infinite.

${ }^{14}$ Note that within this definition, a stationary strategy or the resulting stationary equilibria do not necessarily yield stationary outcomes. Indeed, as we will see below, Equilibrium $I$ potentially produces a quote pattern which does not ressemble a time-invariant stationary process at all.
} 


\subsection{Execution probabilities}

To compute her expected value from LO trading, each trader needs to determine the execution probability corresponding to each possible quote. Focusing on the buyer's problem, let $\Gamma(B)$ be the execution probability of a buy limit order with price $B .{ }^{15}$ Consequently, her corresponding expected utility could be represented as $E(U(B))=\Gamma(B)\left(V_{h}-B\right)$ with $\Gamma(B)$ non-decreasing in $B$. Hence, each buyer faces a trade-off between a better execution price and a higher execution probability. To optimally determine $\Gamma(B)$ and the corresponding bid quote, a buyer needs to account for the market order strategies of future sellers as the execution of a limit order is triggered by the submission of a counterparty market order. Hence, in general, traders' optimal order placement strategies depend on the probability of execution, which in turn is endogenous and is determined by their order placement strategies. For example, consider the problem of a buyer who has to choose a bid. As her order only holds for one period, she only needs to address the seller arriving in the next period. Quoting a high bid, i.e. greater or equal to the cutoff price of the seller (see Proposition 1), will lead to execution of the limit order if the trader arriving next indeed is a seller. Hence, the execution probability at this quote is equal to the probability a seller arrives, i.e. $1-k$. If she quotes a low bid, inferior to the cutoff price of the seller, her execution probability is zero. To determine this cutoff price, the buyer needs to solve the seller's trading problem. As sellers have an expanded trading horizon of two periods, they may position their quote to address only one or both subsequent arriving traders (implying two potential seller cutoff prices arise). Consequently, they not only solve the incoming buyer's trading problem, but also need to account for potential direct competition of subsequently arriving sellers. This will render the nature of this problem somewhat more complex. I will further address this issue in the next section. However, what is clear is that the limit order execution probabilities are endogenous, implying traders are in a game-situation. In the following subsection, the equilibrium of this game is defined.

\subsection{Equilibrium definition}

An equilibrium of the trading game are order placement strategies, $\Sigma($.$) and$ $\Phi($.$) , such that the orders prescribed by the strategies maximize the traders'$ (expected) value from trading when the probability of execution is computed assuming that all traders follow these strategies.

Definition 2 A stationary market equilibrium is a set of strategies $\Sigma^{*}($.$) for$ the buyers and $\Phi^{*}($.$) for the sellers such that for all t \in\{1,2 \ldots,+\infty\}$ :

a.1) $B^{*} \in \operatorname{Arg} \max _{B} E(U(B))$ given $\Sigma^{*}($.$) and \Phi^{*}($.$) ,$

a.2) $B_{0}^{*}=V_{h}-E\left(U\left(B^{*}\right)\right)$;

\footnotetext{
${ }^{15}$ The seller's problem is completely symmetric with $\Psi(A)$ a sell LO's execution probability with price $A$. Further, note that in the absence of a trade, utility is normalized to zero.
} 


$$
\begin{aligned}
& \text { b.1) } A^{*} \in \underset{A}{\operatorname{Argmax}} E(U(A)) \text { given } \Sigma^{*}(.) \text { and } \Phi^{*}(.) \text {, } \\
& \text { b.2) } A_{0}^{*}=V_{l}+E\left(U\left(A^{*}\right)\right) \text {. }
\end{aligned}
$$

Thus, the strategies of all the traders are required to be optimal given the strategies of the other traders. On the one hand, conditions $a .2$ ) and b.2) describe the cutoff-rule-based optimal market order strategies of the buyers and the sellers, respectively (see Proposition 1). Conditions a.1) and b.1), on the other hand, allow to specify the optimal quote (which in turn depends on the market order strategies of the future traders). As there clearly are endogenous linkages between the market and limit order placement strategies, I will determine them simultaneously. ${ }^{16}$

\section{Analysis of the equilibria}

In this section, I will derive two stationary equilibria for this sequential trading process. First, the optimal initial quotes and the existence conditions are determined. Next, both equilibria are formally presented. Finally, in a discussion a further analysis of these equilibria is provided, as well as some insight on the (position of the) market's bid-ask spread.

\subsection{Construction of the equilibria}

\subsubsection{Optimal initial quotes}

Consider a seller arriving in the market and positioned first in line on that side of the market. In case she wants to submit a limit order, she needs to account for future direct competition by the seller potentially arriving in the next period. Doing so, she could opt for two distinct ask quotes. On the one hand, she could submit a limit order at $A_{I}$, which is the highest ask quote at which incoming buyers are willing to submit a market order. This quote, however, will certainly be undercut in case the next arriving trader also is a seller. Hence, it has a low execution probability $(k)$, and thus a high execution risk. ${ }^{17}$ On the other hand, she could submit $A_{I I}$, which is a lower quote that is "undercutting-proof", i.e. no future arriving seller will find it profitable to undercut it. Note that this restriction may cause it to be lower than the buyer's cutoff price. ${ }^{18}$ Hence,

\footnotetext{
${ }^{16}$ Do note this is a Nash Perfect Equilibrium. Actually, the stationarity insures that a trader's strategy is optimal even when she observes prices $(B$ or $A)$ which should not be observed if the players follow their equilibrium strategies (see Foucault (1995)).

${ }^{17}$ Note that despite the presence of risk neutral traders, we use the concept "execution risk" in this analysis. This notion of risk should not be regarded in a traditional economic analysis way (in which the risk neutral trader should not care at all about risk), but rather as a reflection of uncertainty of execution. As such, we follow the standard terminology in the market microstructure literature.

${ }^{18}$ As such, due to the direct competition between sellers, buyers may receive a "bonus" in MO trading. Whether this bonus realizes or not will depend on the market parameters. We will further elaborate on this in the next subsection.
} 
potentially catering to arriving buyers in the two subsequent periods, it has a higher execution probability $(k+(1-k) k)$ and thus a lower execution risk. The choice between $A_{I}$ and $A_{I I}$ will hinge on market parameters and will be further discussed below, as well as the magnitude of both quotes. Submitting a limit order at all other quotes will be sub-optimal for the first-in-line seller:

(i) if $A_{i}>A_{I}$, then the corresponding execution probability $\Psi\left(A_{i}\right)=0$ as $A_{I}$ is the highest ask quote at which incoming buyers are willing submit a $\mathrm{MO}$;

(ii) if $A_{I}>A_{i}>A_{I I}$, the expected value from trading at $A_{i}$ is lower than at $A_{I}$ as $\Psi\left(A_{i}\right)=\Psi\left(A_{I}\right)$;

(iii) if $A_{i}<A_{I I}$, the expected value from trading at $A_{i}$ is lower than at $A_{I I}$ as $\Psi\left(A_{i}\right)=\Psi\left(A_{I I}\right)$.

As an illustration of this quote scheme, in Figure 2 the ask quotes are depicted with their corresponding execution probabilities.

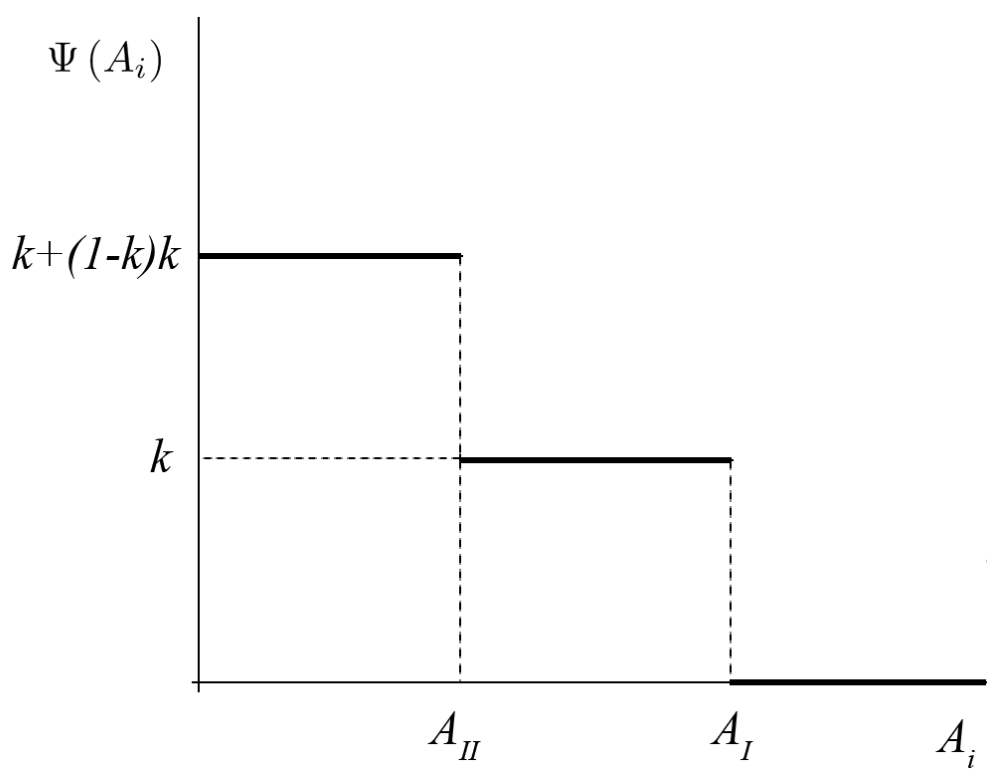

Figure 2: Optimal initial quotes and their corresponding execution probabilities

In contrast, a buyer willing to submit a limit order does not need to account for direct competition by other buyers as her trading horizon only lasts one period. Hence, she will always opt for a limit order at the lowest bid quote at which incoming sellers are willing to submit a market order. Thus, in conformity 
with the equilibrium definition, both buyers and sellers account for each other's actions in determining their optimal quote.

Lemma 1 The optimal bidding strategies for buyers and first-in-line sellers are as follows:

- for a buyer, the optimal bidding strategy is to quote a price equal to the cutoff price of a seller, which in turn depends on the seller's own quoting strategy (see Definition 2). Therefore, given the arrival of a buyer, the submitted quotes in the market can only be:

$$
B_{I}^{*}=A_{0}^{*}\left(A_{I}\right) \text { or } B_{I I}^{*}=A_{0}^{*}\left(A_{I I}\right) ;
$$

- for a first-in-line seller, the optimal bidding strategy is to quote a price at most equal to the cutoff price of a buyer, which in turn depends on the buyer's own quoting strategy (see Definition 2). Therefore, given the arrival of a first-in-line seller, the submitted quotes in the market can only be:

$$
A_{I}^{*}=B_{0}^{*}\left(B_{I}\right) \text { or } A_{I I}^{*} \leq B_{0}^{*}\left(B_{I I}\right) .
$$

Proof. Contained in the discussion above.

\subsubsection{Existence conditions}

Focusing on the quote choice by first-in-line traders, overall, one could discern two types of equilibria:

- In the first equilibrium ( $I$ ), all the first-in-line sellers choose to run a high execution risk and quote $A_{I}$. In this case, the expected value of submitting a limit sell order is: $E\left(U\left(A_{I}\right)\right)=k\left(A_{I}-V_{l}\right)$. In fact, the seller could run the chance of a better execution by quoting $A_{I I}$ instead, rendering an expected value of: $E\left(U\left(A_{I I}\right)\right)=(k+(1-k) k)\left(A_{I I}-V_{l}\right)$. So, for this first equilibrium to exist, the following condition must be satisfied for all the sellers: $E\left(U\left(A_{I}\right)\right)>E\left(U\left(A_{I I}\right)\right)$ or $k\left(A_{I}-V_{l}\right)>(k+(1-k) k)\left(A_{I I}-V_{l}\right)$. Buyers, which do not have to account for future direct competition from other buyers, always opt for the lowest bid (i.e., $B_{I}$ ) at which the incoming seller (quoting $A_{I}$ as it always is a first-in-line seller) is indifferent between a MO and a LO: $B_{I}=V_{l}+k\left(A_{I}-V_{l}\right){ }^{19}$

- In contrast with the first equilibrium, in the second equilibrium (II) all first-in-line sellers choose to run a low execution risk and quote $A_{I I}$. In this case, the existence conditions are: $k\left(A_{I}-V_{l}\right)<(k+(1-k) k)\left(A_{I I}-V_{l}\right)$ and $B_{I I}=V_{l}+(k+(1-k) k)\left(A_{I I}-V_{l}\right)$.

\footnotetext{
${ }^{19}$ In fact, for the buyers, the following inequality must be satisfied in equilibrium: $E\left(U\left(B_{I}\right)\right)>E\left(U\left(B_{I I}\right)\right)$, which is always the case as $B_{I I}$ is too low to attract sellers quoting $A_{I}$ causing $\Gamma\left(B_{I I}\right)=0$.
} 
Clearly, the arrival of a one-period-horizon buyer, always "resets" the limit order book state as a seller arriving in the subsequent period will always be firstin-line. Hence, in this case there is always a return to the initial state of either Equilibrium $I$ or Equilibrium II, depending on the market parameters. However, the arrival of consecutive sellers might lead to undercutting of the initial ask price $A_{I}$ in Equilibrium $I$, as anticipated by the sellers in the determination of their execution probability. This undercutting will occur as follows:

As we are in Equilibrium $I$, a seller arriving when the LOB is empty will always quote $A_{I}$. A second seller arriving in the subsequent period will have the choice to join the existing queue at $A_{I}$ (rendering an expected payoff of $k^{2}\left(A_{I}-V_{l}\right)$ ) or to undercut $A_{I}$ by one tick (rendering $\left.k\left(A_{I}-\Delta-V_{l}\right)\right) .{ }^{20}$ Hence, she will only have an incentive to undercut $A_{I}$ if $k^{2}\left(A_{I}-V_{l}\right)<k\left(A_{I}-\Delta-V_{l}\right)$. The arrival of consecutive sellers in subsequent periods causes this quote to be undercut even further as long as the following conditions hold:

(i) $k^{2}\left(A_{i}-V_{l}\right)<k\left(A_{i}-\Delta-V_{l}\right)$;

(ii) $k^{2}\left(A_{I}-V_{l}\right)<k\left(A_{i}-\Delta-V_{l}\right)$;

(iii) $(k+(1-k) k)\left(A_{I I}-V_{l}\right)<k\left(A_{i}-\Delta-V_{l}\right)$.

These conditions hold when undercutting by one tick is more profitable than (i) joining the queue, (ii) returning to $A_{I}$, or (iii) immediately shifting down to $A_{I I} \cdot{ }^{21}$ When a certain quote $\tilde{A}$ is reached, within this particular Equilibrium $I$ the condition stated last no longer holds, i.e. now $(k+(1-k) k)\left(A_{I I}-V_{l}\right)>k\left(\tilde{A}-\Delta-V_{l}\right)$, whereas the other two conditions are still valid. Hence, undercutting $\tilde{A}$ by one tick is now less profitable than immediately dropping down to $A_{I I}$. Finally, given this quote $A_{I I}$, an arriving seller could either revert to $A_{I}$ or join the queue at $A_{I I}$, which is denoted by " $R$ " and " $N R$ ", respectively. This choice will depend on the direction of the inequality sign in $k^{2}\left(A_{I}-V_{l}\right) \gtrless\left(k^{2}+(1-k) k\right)\left(A_{I I}-V_{l}\right)$.

Note that conditions (i) and (ii) cannot reverse as long as condition (iii) still holds. Hence, before stabilizing at a certain level or reverting to $A_{I}$, the quote always drops to the undercutting-proof quote due to the higher execution probability this quote offers. For instance, should condition (i) reverse while condition (iii) still holds, then the quote at which this occurs suddenly becomes the lowest possible quote as all sellers start joining the queue at that quote. Consequently, as it will never be undercut anymore, in fact the undercuttingproof quote $A_{I I}$ is reached, inherently implying condition (iii) is also reversed.

\footnotetext{
${ }^{20}$ Undercutting occurs tick by tick as this strategy always produces the highest quote rendering an execution probability of $k$.

${ }^{21}$ Note that, in fact, condition (ii) fully encompasses condition (i) as $k^{2}\left(A_{I}-V_{l}\right) \geq k^{2}\left(A_{i}-\right.$ $\left.V_{l}\right)$. Hence, merely joining the queue can never be an optimal strategy. We, however, keep condition (i) listed here for illustrational purposes (i.e., to distinguish the reverting and the non-reverting version of Equilibrium $I$ (see below)).
} 
In this case the non-reverting version of Equilibrium $I$, i.e. $I-N R$, is obtained. Alternatively, should condition (ii) reverse while condition (iii) still holds, then further tick-by-tick undercutting becomes less profitable than reverting to $A_{I}$. However, the next seller will undercut once more to the quote that offers a high execution probability $k+(1-k) k$ and at which trading is still more profitable than at $A_{I}$, i.e. at the undercutting-proof quote in this equilibrium at which $k^{2}\left(A_{I}-V_{l}\right)<(k+(1-k) k)\left(A_{I I}-V_{l}\right)$. Hence, again condition (iii) is also reversed and the reverting version of Equilibrium $I$, i.e. $I-R$, is obtained. Thus, the variable undercutting-proof quote $A_{I I}$ in this equilibrium is positioned to account for the reversion of conditions ( $i$ ) and (ii) (see below).

Each of these possibilities, as well as Equilibrium $I I$ will be discussed in the next subsection.

\subsection{Equilibria}

\subsubsection{Equilibrium I}

As first-in-line sellers only target buyers arriving in the consecutive period in this equilibrium, these quotes are completely similar to those described in Foucault (1995, 1999) and Handa, Schwartz and Tiwari (2003) in which all traders have a single-period horizon.

Proposition 2 EQUILIBRIUM I:

Define $\mu=\frac{k}{1-(1-k) k}$ and $\lambda=\frac{1-k}{1-(1-k) k}$. If $\mu\left(V_{h}-V_{l}\right)-(2-k)\left(M-V_{l}\right)>$ 0 , then an equilibrium in which all first-in-line sellers choose to run a high execution risk exists. In equilibrium, they always submit a market order if there is a LO buy quote available at:

$$
B_{I}^{*}=\lambda V_{l}+(1-\lambda) V_{h}=(1-\lambda)\left(V_{h}-V_{l}\right)+V_{l}
$$

Otherwise, they quote an ask equal to:

$$
A_{I}^{*}=\mu V_{h}+(1-\mu) V_{l}=\mu\left(V_{h}-V_{l}\right)+V_{l},
$$

where:

$$
M=V_{l}+\min \left[\frac{k}{1-2 k+3 k^{2}-k^{3}}\left(V_{h}-V_{l}\right) ; \max \left(\Delta \frac{2-k}{1-k} ; \frac{k \mu}{(2-k)}\left(V_{h}-V_{l}\right)+\Delta\right)\right] .
$$

Proof. See Appendix.

Both quotes are shown to depend upon (1) the difference in traders' valuations, (2) the proportion of buyers and sellers, and (3) the level of the seller's private valuation. $B_{I}^{*}$ could be seen as a weighted combination of $V_{l}$ (i.e., the reservation value of a market order seller) and $V_{h}$ (i.e., the reservation value of a limit order buyer), where the weights are given by $\lambda$ and $1-\lambda$, respectively. Hence, $\lambda$ determines the location of $B_{I}^{*}$ between $V_{l}$ and $V_{h}$ and thus how the benefits of trading are shared between the limit order buyer quoting $B_{I}^{*}$ and the 
market order seller that subsequently hits that quote. If $\lambda \rightarrow 1, B_{I}^{*}$ approaches $V_{l}$ and the market order seller's gain from trading goes to zero. Conversely, if $\lambda$ $\rightarrow 0, B_{I}^{*}$ approaches $V_{h}$ and the limit order buyer's expected gain from trading goes to zero. More specifically, the term $\lambda$ is the ratio of the complement of two probabilities: (1) the unconditional probability of a buyer arriving in the market place $k$, and (2) the joint probability that a buyer and a seller arrive sequentially in this order $(1-k) k$ (which is necessary for a limit buy order to be hit by a market sell order). As such, $\lambda$ reflects the relative risk of non-execution to a buyer vis-à-vis the risk of non-execution to both parties. The limit order buyer optimally places the bid to apportion the gain from trading in accordance with this ratio. Within this equilibrium, the intuition behind the optimal ask price, $A_{I}^{*}$, and the parameter $\mu$, is completely symmetric. Clearly, both quotes are "shadow quotes" as only one of the two can be observed at any time.

Equilibrium path ask quote Whereas the initial bid quote $B_{I}$ is fixed throughout time, the arrival of consecutive sellers could potentially lead to undercutting of the initial ask price $A_{I}$ in Equilibrium $I$, as anticipated by the sellers in the determination of their execution probability. Two potential undercutting paths for the ask quote are considered.

In a first path, consecutively arriving sellers undercut $A_{I}$ tick-by-tick until a certain quote $\tilde{A}$ is reached. At this quote undercutting by one tick becomes less profitable than immediately dropping down to the undercutting-proof quote $A_{I I}$. Given this quote $A_{I I}$, an arriving seller will opt to revert to $A_{I}$ as this renders the highest payoff. Proposition 3 formalizes this equilibrium path, while Figure 3 depicts it.

Proposition 3 EQUILIBRIUM I-R:

When: $\quad \mu\left(V_{h}-V_{l}\right)-(2-k)\left(M-V_{l}\right)>0$,

$$
k \mu\left(V_{h}-V_{l}\right)-(2-k)\left(M-V_{l}\right)+\Delta<0,
$$$$
\Delta>0 \text {, }
$$$$
k \mu\left(V_{h}-V_{l}\right)-M+V_{l}>0,
$$

then, in equilibrium, all sellers quote:

1. AI given an empty $L O B$,

2. $A_{i}-\Delta$ when the current best quote is $A_{i}$ lying within $\left(\tilde{A}+\Delta, A_{I}\right)$,

3. $A_{I I}$ when the current best quote is lying within $\left[A_{I I}, \tilde{A}+\Delta\right]$,

4. $A_{I}$ when the current best quote is $A_{I I}$, 
where $\tilde{A}=(2-k)\left(M-V_{l}\right)+V_{l}$ is the quote at which undercutting by one tick is no longer the most profitable strategy (i.e., where it becomes more profitable to drop immediately to $\left.A_{I I}\right)$.

Proof. See Appendix.

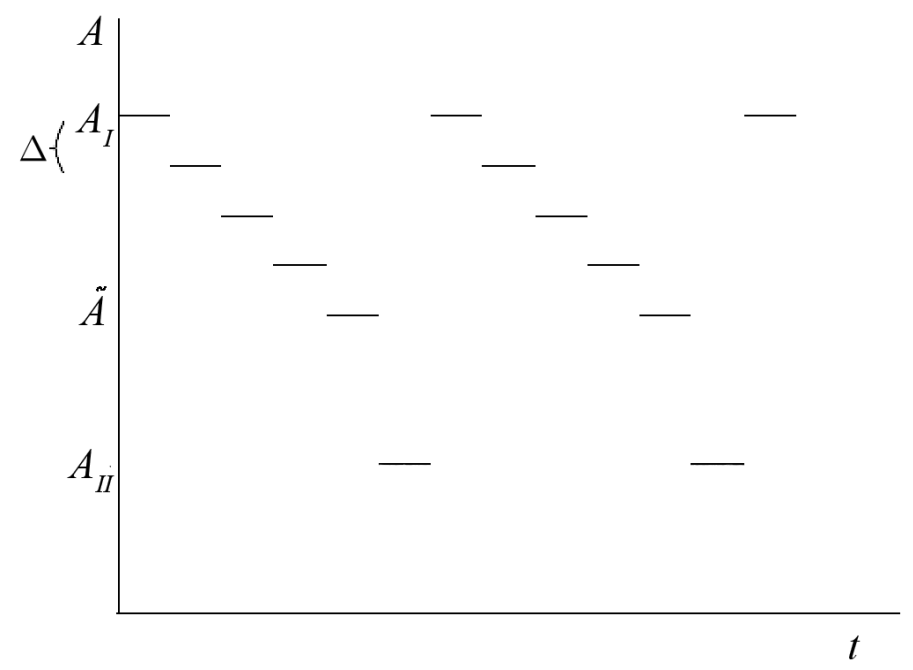

Figure 3: Ask quote equilibrium path within Equilibrium I-R

Note that the generated path is in fact very similar to the Edgeworth cycle ${ }^{22}$ observed by Maskin and Tirole (1988) when modeling price competition within a dynamic oligopoly (in which firms take turns choosing prices). In this Markov Perfect Equilibrium $(\mathrm{MPE})^{23}$, to increase their market share firms undercut each other successively ("price war phase") until the price reaches the competitive level (i.e., where it equals marginal cost), at which point a "war of attrition" starts ("relenting phase") in which each firm waits for one of the others to raise price (i.e., relent). ${ }^{24}$ Finally, when sticking to the low price and consequently making no profits becomes too costly, this will realize (i.e., some firm reverts to the initial high price), after which price cutting begins again. As in our model,

\footnotetext{
${ }^{22}$ Note that Edgeworth (1925) developed the model producing this cycle as a dynamic attempt to improve upon the static Bertrand (1883) model (which always yielded the competitive price as an outcome, while in reality typically $\mathrm{p}>\mathrm{MC}$ with few sellers). Another alternative to overcome this faillure is the "kinded demand curve".

${ }^{23}$ Note that an MPE entails that a firm's strategy in any period is assumed only to depend on the currently observed price (i.e., the payoff-relevant state).

${ }^{24}$ The act of relenting is related to its public good nature from the firms' point of view. All firms wish to raise their prices, but each would like the others to raise price first so as to be able to undercut. Maskin and Tirole (1988) argue that mixed strategies, where each firm relents with probability less than one, are quite natural as a resolution to this free-rider problem.
} 
during the price war phase, a firm undercuts because it does not trust the others. Sticking to the high price/quote will not prevent the other firms/traders from being aggressive. As such, Maskin and Tirole argue "mistrust is a self-justifying attitude".

Relatedly, within the market microstructure literature, Cordella and Foucault (1999) model the dynamic competition between two dealers to capture the real-world sequential bidding process instead of applying Bertrand price competition models with simultaneous decision making to formalize this bidding process, as was typically done before. As such, they are able to address the question of how best quotes evolve in between transactions. When the price in the market is above the competitive price, they argue a dealer faces the following choice (similar to the LO trader's trade-off in this paper): either he just posts the competitive price (which secures execution of the next incoming market order at a low profit), or he undercuts the current quote by only one tick (which renders a larger profit, but does not guarantee execution ${ }^{25}$ ). When time priority is enforced, Cordella and Foucault (1999) argue dealers keep undercutting each other until one of them eventually posts the competitive price. As such, the best quote in the market converges to the competitive price where the speed of convergence hinges on the frequency with which dealers check their offers. This quote pattern contrasts those observed in Maskin and Tirole (1988) and in Equilibrium $I-R$ of the current model. It does resemble, however, the path observed in Equilibrium $I-N R$, which will be discussed now.

A second path is completely similar to the first path, except for the reversion when quote $A_{I I}$ is reached. Instead, in this path, when $A_{I I}$ is reached, future consecutively arriving sellers will tend to join the queue at this quote. ${ }^{26}$ Proposition 4 formalizes this equilibrium path, while Figure 4 depicts it.

Proposition 4 EQUILIBRIUM I - NR:

When

$$
\begin{aligned}
& \mu\left(V_{h}-V_{l}\right)-(2-k)\left(M-V_{l}\right)>0, \\
& k \mu\left(V_{h}-V_{l}\right)-(2-k)\left(M-V_{l}\right)+\Delta<0, \\
& \Delta>0, \\
& k \mu\left(V_{h}-V_{l}\right)-\left(M-V_{l}\right)<0,
\end{aligned}
$$

then, in equilibrium, all sellers quote:

1. $A_{I}$ given an empty $L O B$,

2. $A_{i}-\Delta$ when the current best quote is $A_{i}$ lying within $\left(\tilde{A}+\Delta, A_{I}\right)$,

\footnotetext{
${ }^{25}$ In their model, this large profit realizes when a market order arrives before the competitordealer is able to undercut this quote. Clearly, the time of arrival of market orders is unknown to dealers. The size of the execution risk hinges on the reaction speed of the competitor.

${ }^{26} \mathrm{As}$ argued before, this is due the fact that for these sellers the higher execution probability corresponding to $A_{I I}$ (i.e., $k$ vs. $k^{2}$ at $A_{I}$ ) outweighs the quote advantage related to switching from $A_{I I}$ to $A_{I}$.
} 
3. $A_{I I}$ when the current best quote is lying within $\left[A_{I I}, \tilde{A}+\Delta\right]$,

4. $A_{I I}$ when the current best quote is $A_{I I}$,

where $\tilde{A}=(2-k)\left(M-V_{l}\right)+V_{l}$ is the quote at which undercutting by one tick is no longer the most profitable strategy (i.e., where it becomes more profitable to drop immediately to $\left.A_{I I}\right)$.

Proof. See Appendix.

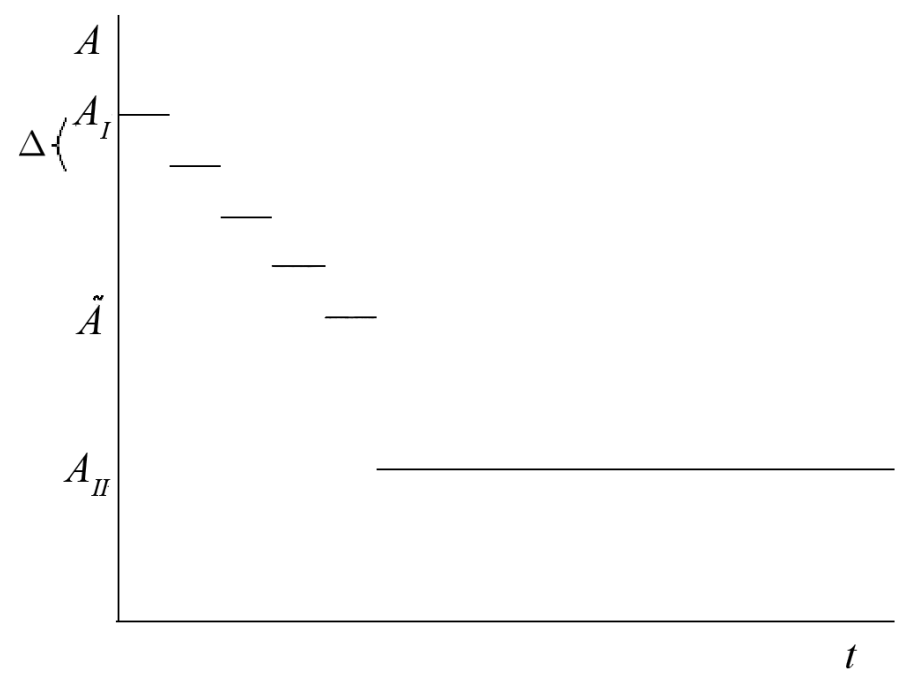

Figure 4: Ask quote equilibrium path within Equilibrium I-NR

Equilibrium path bid quote Consecutively arriving buyers will always quote the equilibrium quote $B_{I}$, thus trying to induce the incoming seller to submit a MO.

Proposition 5 EQUILIBRIUM I:

When $\mu\left(V_{h}-V_{l}\right)-(2-k)\left(M-V_{l}\right)>0$,

then, in equilibrium, all buyers quote:

1. $B_{I}$ given any state of the order book.

Proof. See Appendix. 


\subsubsection{Equilibrium II}

For a different set of parameters, another equilibrium with different properties is obtained.

Proposition 6 EQUILIBRIUM II:

If $\frac{k}{1-(1-k) k}\left(V_{h}-V_{l}\right)-(2-k)\left(M-V_{l}\right)<0$, then an equilibrium in which all first-in-line sellers choose to run a low execution risk exists. In equilibrium, they always submit a market order if there is a LO buy quote available at:

$$
B_{I I}^{*}=V_{l}+(k+(1-k) k)\left(M-V_{l}\right) ;
$$

Otherwise, they quote an ask equal to:

$$
A_{I I}^{*}=M,
$$

where:

$M=V_{l}+\min \left[\frac{k}{1-2 k+3 k^{2}-k^{3}}\left(V_{h}-V_{l}\right) ; \max \left(\Delta \frac{2-k}{1-k} ; \frac{k \mu}{\mu(2-k)}\left(V_{h}-V_{l}\right)+\Delta\right)\right]$.

Proof. See Appendix.

The $A_{I I}$-quote should always be positioned accounting for the actions of potential counterparties:

(i) incoming buyers at least need to be indifferent between submitting a $\mathrm{MO}$ at $A_{I I}$ or a $\mathrm{LO}$ at their own $B_{I I}: V_{h}-A_{I I} \geq(1-k)\left(V_{h}-B_{I I}\right)$, which could be rewritten as $A_{I I}^{*} \leq \frac{k}{1-2 k+3 k^{2}-k^{3}}\left(V_{h}-V_{l}\right)+V_{l}$.

Further, it should also account for the action of the seller potentially arriving in the subsequent period. Hence, it should comply to at least one of the following undercutting-proof conditions (i.e., the one that is least binding):

(ii) for the seller arriving in the subsequent period undercutting $A_{I I}$ by one tick renders at most the payoff of joining the queue at $A_{I I}:(k+(1-$ $k) k)\left(A_{I I}-\Delta-V_{l}\right) \leq\left(k^{2}+(1-k) k\right)\left(A_{I I}-V_{l}\right)$, which could be rewritten as $A_{I I} \leq \Delta \frac{2-k}{1-k}+V_{l}$;

(iii) for the seller arriving in the subsequent period undercutting $A_{I I}$ by one tick renders at most the payoff of submitting a LO at $A_{I}:(k+(1-$ $k) k)\left(A_{I I}-\Delta-V_{l}\right) \leq k^{2}\left(A_{I}-V_{l}\right)$, which could be rewritten as $A_{I I} \leq$ $\frac{k \mu}{(2-k)}\left(V_{h}-V_{l}\right)+\Delta+V_{l}$.

The minimax-rule within the definition of $M$ positions the $A_{I I}$-quote in accordance to these boundary conditions. Typically, for low values of $V_{h}-V_{l}$, the indifference condition (i) is most binding. For intermediate values of $V_{h}-V_{l}$, undercutting-proof condition (ii) solves $M$ and thus provides the optimal $A_{I I}$. Finally, for high values of $V_{h}-V_{l}$, undercutting-proof condition (iii) renders the optimal $A_{I I}$. All three possibilities are presented in Figure 5. For illustrational purposes the intercept $V_{l}$ is normalized to zero, $k=\frac{1}{2}$ and $\Delta=1$ which renders $M=\min \left[\frac{4}{5} V_{h} ; \max \left(3 ; \frac{2}{9} V_{h}+1\right)\right]$. The solution to this minimax-problem is depicted in bold in this figure. 


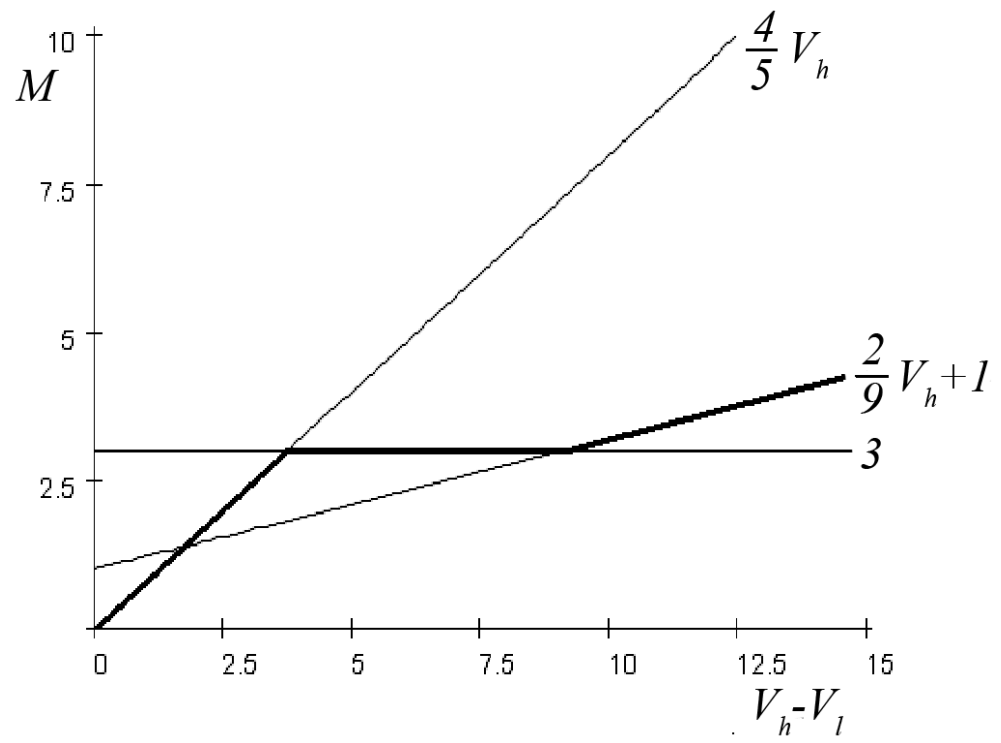

Note: Within this graph $k=\frac{1}{2}, \Delta=1$ and the intercept $V_{l}$ is normalized to zero.

Figure 5: Illustration of the initial ask quote within Equilibrium II

Equilibrium path ask quote Over time, within this equilibrium, consecutively arriving sellers tend to join the queue at $A_{I I} \cdot{ }^{27}$ It is clear that although the quoting behavior is very similar over time, trading gains are not, as the payoff of the queue-joining seller is always somewhat lower due to the lower probability of execution (i.e., $k+(1-k) k$ versus $\left.k^{2}+(1-k) k\right){ }^{28}$

\section{Proposition 7 EQUILIBRIUM II:}

When $\mu\left(V_{h}-V_{l}\right)-(2-k)\left(M-V_{l}\right)<0$,

$$
k \mu\left(V_{h}-V_{l}\right)-\left(M-V_{l}\right)<0,
$$

then, in equilibrium, all sellers quote:

\section{AII given any state of the order book.}

Proof. See Appendix.

\footnotetext{
${ }^{27}$ This equilibrium path corresponds to the setting where restriction (ii) is less binding than restriction (iii). As will be shown in the next subsection, in case restriction (iii) is the least binding one, Equilibrium $I I$ always appears to be fully dominated by Equilibrium $I$. Resultingly, an ask quote equilibrium path which alters between $A_{I I}$ and $A_{I}$ never realizes in practice.

${ }^{28}$ Note that this difference becomes arbitrarily small when $k \rightarrow 1$.
} 
Equilibrium path bid quote Consecutively arriving buyers will always quote the equilibrium quote $B_{I I}$, thus trying to induce the incoming first-in-line seller to submit a MO.

Proposition 8 EQUILIBRIUM II:

When $\mu\left(V_{h}-V_{l}\right)-(2-k)\left(M-V_{l}\right)<0$,

then, in equilibrium, all buyers quote:

1. $B_{I I}$ given any state of the order book.

Proof. See Appendix.

\subsection{Discussion}

Within this subsection, I will first discuss the existence and the properties of the bid-ask spread. Next, the positioning of the different equilibria is highlighted. Further, a parametrization of the model is provided. Finally, expected trading gains for both trader types are analyzed.

\subsubsection{Properties of the bid-ask spread}

The initial equilibrium quotes are positioned such that the market is able to clear for all values of $k$. A seller that submits a LO at $A_{I}$ or $A_{I I}$ implicitly accounts for the associated non-execution risk and sets the LO's expected utility equal to that of immediately transacting via a $\mathrm{MO}$ at $B_{I}$ or $B_{I I}$, respectively. The buyer's perspective is symmetric (although her non-execution risk is fixed for both quotes). Hence, at these quote levels, which are positioned in such a way that all traders arriving in the market are satisfied ex ante and have no ex post regret, participants are willing to take the risk of submitting a LO. It is precisely this willingness that enables the market to clear for all values of $k$. Specifically, when there is an imbalance (i.e., $k<\frac{1}{2}$ or $k>\frac{1}{2}$ ), a rationing problem arises and no single price exists that completely clears the market. Consequently, some LO traders on the heavy side of the market will not be able to fulfil their transaction. ${ }^{29}$ Hence, the difference between both quotes (i.e., the bid-ask spread) compensates these traders' cost of non-execution. For instance, for values of $k$ close to unity, buyers outnumber sellers by far in the market. Due to the indirect intertemporal competition that exists between a buyer today and future buyers, sellers are granted greater market power than in the $k=\frac{1}{2}$ case. In order to induce sellers to trade via a $\mathrm{MO}$, buyers need to quote an attractively high bid quote (close to $V_{h}$ ). Conversely, sellers submitting a LO post their asks aggressively close to $V_{h}$ as they realize buyers rather prefer to trade via a MO. Consequently, in both equilibria $I$ and $I I$ the bid and ask quotes are positioned close to $V_{h}$ and, as will be made clear below, the spread is

\footnotetext{
${ }^{29}$ Note that these traders have been selected by chance rather than by an explicit rationing device.
} 
quite tight. Analogously, for values of $k$ close to zero, both quotes are positioned close to $V_{l}$ with a relatively tight spread. This result is reminiscent of Foucault (1995, 1999) and Handa et al. (2003). Do note that within this model, this spread is the mere result of strategic rent-seeking by liquidity-supplying limit order traders. ${ }^{30}$

Proposition 9 A natural positive virtual initial spread exists in the market that is given by: ${ }^{31}$

$$
\begin{aligned}
& \text { (i) } S_{I I}=(1-(k+(1-k) k))\left(\frac{k}{1-2 k+3 k^{2}-k^{3}}\left(V_{h}-V_{l}\right)\right)=\frac{k(1-k)^{2}}{1-2 k+3 k^{2}-k^{3}}\left(V_{h}-V_{l}\right) \\
& \text { if } A_{I I}^{*}=\frac{k}{1-2 k+3 k^{2}-k^{3}}\left(V_{h}-V_{l}\right)+V_{l}, \\
& \text { (ii) } S_{I I}=(1-(k+(1-k) k)) \Delta \frac{2-k}{1-k}=(1-k)(2-k) \Delta \\
& \text { if } A_{I I}^{*}=\Delta \frac{2-k}{1-k}+V_{l}, \\
& \text { (iii) } S_{I}=(1-k) \frac{k}{1-(1-k) k}\left(V_{h}-V_{l}\right)=(1-k) \mu\left(V_{h}-V_{l}\right) \\
& \text { if } A_{I}^{*}=\mu\left(V_{h}-V_{l}\right)+V_{l},
\end{aligned}
$$

for low, intermediate and high values of $V_{h}-V_{l}$, respectively. ${ }^{32}$

Proof. See Appendix.

For both equilibria, the spread is always composed as the product of the complement of the seller's execution probability within the considered equilibrium, and the seller's added value of the quote in excess of her own private valuation $V_{l}$. It is "virtual" by nature, as both quotes will never be observed simultaneously in the market due to the structure of the model. ${ }^{33}$ The term "initial" indicates that the quotes constituting it will only be observed upon the arrival of a buyer or a first-in-line seller. More information on the shape of these spread functions is provided in the proof of Proposition 9 in the Appendix.

\subsubsection{Positioning of the different equilibria}

Figure 6 depicts for each value of $k$, the sets of values of $V_{h}-V_{l}$ for which the different equilibria exist (with $\Delta$ set equal to 1 ). In deriving this graph, implicitly the initially stated parameter restrictions, i.e. $V_{l}>0, V_{h}>0, V_{h}-$

\footnotetext{
${ }^{30}$ Thus, a spread exists in this order driven market merely due to a difference in traders' valuations, even in the absence of inventory costs and asymmetric information.

${ }^{31}$ The term "natural" reflects the fact that this spread arises in the model without the presence of frictions like for instance asymmetrically informed traders, as was argued before. As a benchmark of the importance of non-informational frictions, note that Huang and Stoll (1997) estimate that they on average correspond to $88.8 \%$ of the bid-ask spread.

${ }^{32}$ Note that $S_{I I}$ if $M=\frac{k^{2}}{(1-(1-k) k)(2-k)}\left(V_{h}-V_{l}\right)+\Delta+V_{l}$ was not added as in practice this equilibrium quote will never be posted by first-in-line LO sellers as it is strictly dominated by $A_{I}$ (see below). However, this quote could potentially arise as the final value of the undercutting process.

${ }^{33}$ Hence, they are mere shadow quotes.
} 
$V_{l}>0, k \in(0,1)$, are accounted for to verify which existence conditions are satisfied. At low levels of $V_{h}-V_{l}$, Equilibrium $I I$ holds for all values of $k$. Apparently, the payoffs of trading at the undercutting-proof quotes are higher than those at the Equilibrium $I$ quotes within this low total trading gains zone. ${ }^{34}$ This is the case until the inequality sign in the existence condition separating Equilibria $I$ and $I I$ switches direction on the solid line, and Equilibrium $I-N R$ becomes valid. Hence, the bottom (solid) line represents the value of $V_{h}-V_{l}$ for each $k$ at which sellers are indifferent between Equilibria $I$ and $I I$. On this line, the specific existence condition separating both equilibria holds with equality (i.e., $\left.k\left(A_{I}-V_{l}\right)=(k+(1-k) k)\left(A_{I I}-V_{l}\right) \Rightarrow \mu\left(V_{h}-V_{l}\right)-(2-k)\left(M-V_{l}\right)=0\right)$ which always occurs in the $V_{h}-V_{l}$-region where $M=V_{l}+\Delta \frac{2-k}{1-k}$ (i.e., where the subsequent arriving seller will not undercut but join the queue at $A_{I I}$ ). Below it, Equilibrium $I I$ holds, above, Equilibrium $I$. At very low levels of $k$, it takes a higher $V_{h}-V_{l}$ value for the inequality sign to switch direction. Hence, as most arriving traders are sellers at these levels of $k$, sellers account for this higher intertemporal competition by submitting a low execution risk quote, even when the gains from trading might be fairly high. Within Equilibrium $I$, the upper (dashed) line represents the value of $V_{h}-V_{l}$ for each $k$ at which sellers are indifferent between Equilibria $I-N R$ and $I-R$. Thus, on this line the specific existence condition separating both equilibria holds with equality: $k^{2}\left(A_{I}-V_{l}\right)=\left(k^{2}+(1-k) k\right)\left(A_{I I}-V_{l}\right) \Rightarrow k \mu\left(V_{h}-V_{l}\right)-\left(M-V_{l}\right)=0$, which in fact always occurs at that position in the $V_{h}-V_{l}$-region where traders are indifferent between $M=V_{l}+\Delta \frac{2-k}{1-k}$ and $M=\frac{k \mu}{(2-k)}\left(V_{h}-V_{l}\right)+\Delta+V_{l}$ (i.e., where the arriving seller will not undercut but is indifferent between joining the queue at $A_{I I}$ and reverting to $\left.A_{I}\right)$. Hence, in practice, equilibrium quote $A_{I I}=\frac{k \mu}{(2-k)}\left(V_{h}-V_{l}\right)+\Delta+V_{l}$ will never be observed as it is strictly dominated by $A_{I}$. Below this line, Equilibrium $I-N R$ holds, above, Equilibrium $I-R$. Note that for very high $k$-values both lines start to coincide, implying Equilibrium $I-N R$ does not occur within this region when most arriving traders are buyers. For $k=0$ and $k=1$, both curves asymptotically go to $+\infty$.

To obtain further insight into the positioning of these equilibria, let us briefly reconsider the seller's order submission decision. Upon arrival, each limit order seller has two options: either she submits a high execution risk / high margin quote $\left(A_{I}\right)$, or either she posts a low execution risk / low margin quote $\left(A_{I I}\right)$. To decide upon the preferred option she will compare expected utilities: i.e., $E\left(U\left(A_{I}\right)\right)=\Psi\left(A_{I}\right)\left(A_{I}-V_{l}\right)$ versus $E\left(U\left(A_{I I}\right)\right)=\Psi\left(A_{I I}\right)\left(A_{I I}-V_{l}\right)$. Hence, when deciding to shift from $A_{I}$ to $A_{I I}$, a trader will make the trade-off between the execution probability increase $\Psi\left(A_{I I}\right)-\Psi\left(A_{I}\right)$ and the quote decrease $A_{I I}-$ $A_{I}$.

On the one hand, the execution probability increase always equals $(1-k) k$, which reaches its maximum value of $\frac{1}{4}$ at $k=\frac{1}{2}$. This function is depicted in Figure 7. However, to judge the importance of this increase, it should be

\footnotetext{
${ }^{34}$ Note that for extremely low $V_{h}-V_{l}$ values, it even holds that the undercutting-proof quotes are both positioned at higher levels than the Equilibrium $I$ quotes, which clearly renders a higher payoff (see below).
} 


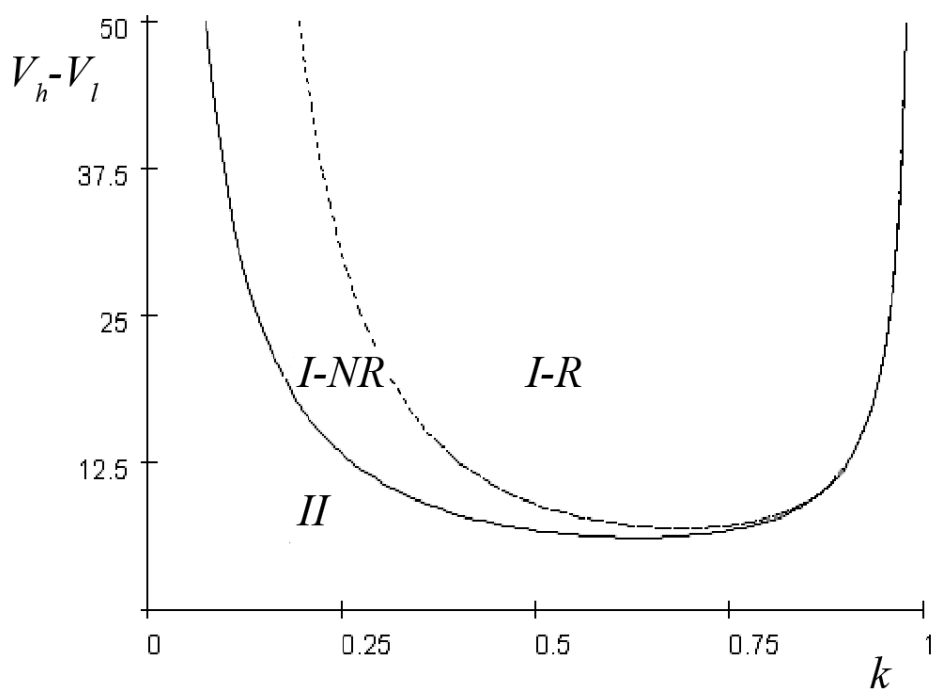

Figure 6: The different equilibria

considered in relative terms (i.e., vis-à-vis the maximal probability of execution $k+(1-k) k)$. In Figure $8, \frac{(1-k) k}{k+(1-k) k}$ is therefore drawn. ${ }^{35}$ Clearly, for lower values of $k$, the increase is relatively more important. Being able to expose a limit order for two periods instead of one becomes a very valuable feature if counterparty buyers are not abundantly present. Consequently, within the low region of $k$, ceteris paribus, due to the elevated direct competition LO sellers are more inclined to quote $A_{I I}$ as compared to other regions. In contrast, within the higher $k$ region, the increase becomes relatively less important. Hence, the added value of going for order exposure in both periods is very low. In this case, the seller has little interest in protecting herself from the (low) direct competition from a consecutively arriving seller and the resulting undercutting of her quote, inducing her to quote $A_{I}$ instead of $A_{I I}$, ceteris paribus.

On the other hand, the quote decrease $A_{I I}-A_{I}$ depends on the values of $k$, $\Delta$ and of $V_{h}-V_{l}:{ }^{36}$

(i) for low values of $V_{h}-V_{l}:\left(\frac{k}{1-2 k+3 k^{2}-k^{3}}-\frac{k}{1-(1-k) k}\right)\left(V_{h}-V_{l}\right)$;

(ii) for intermediate values of $V_{h}-V_{l}: \Delta \frac{2-k}{1-k}-\frac{k}{1-(1-k) k}\left(V_{h}-V_{l}\right)$;

(iii) for high values of $V_{h}-V_{l}:\left(\frac{k^{2}}{(1-(1-k) k)(2-k)}-\frac{k}{1-(1-k) k}\right)\left(V_{h}-V_{l}\right)+\Delta$.

\footnotetext{
${ }^{35}$ In fact, this graph illustrates the effect on the probability of execution of increasing the order exposure from one to two periods.

${ }^{36}$ Note that whereas $A_{I}$ is fixed, $A_{I I}$ depends on the level of $V_{h}-V_{l}$.
} 


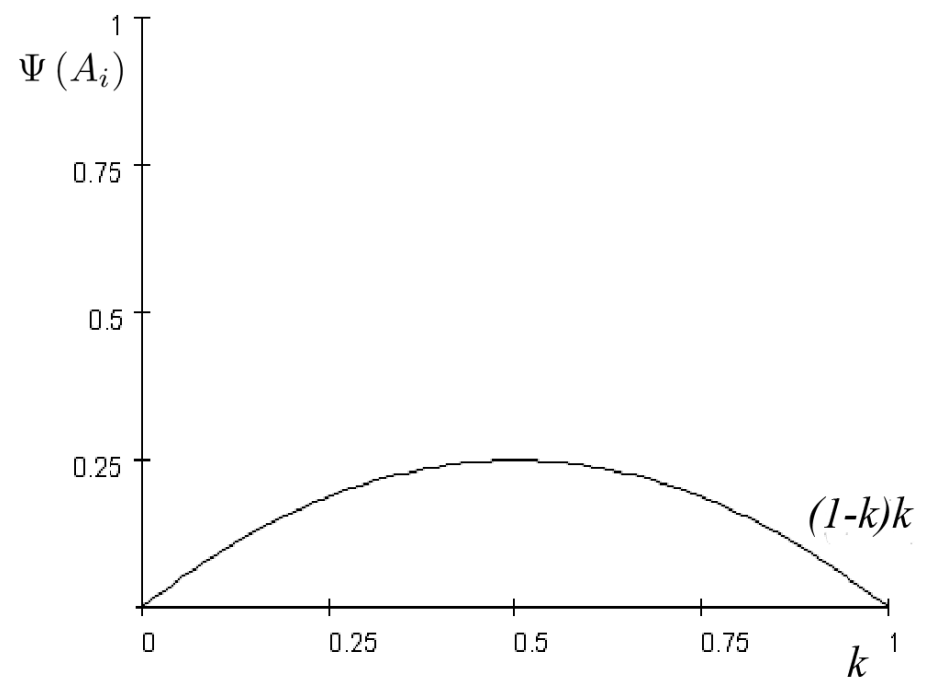

Figure 7: Maximum probability of execution of a limit sell order over a range of $\mathrm{k}$

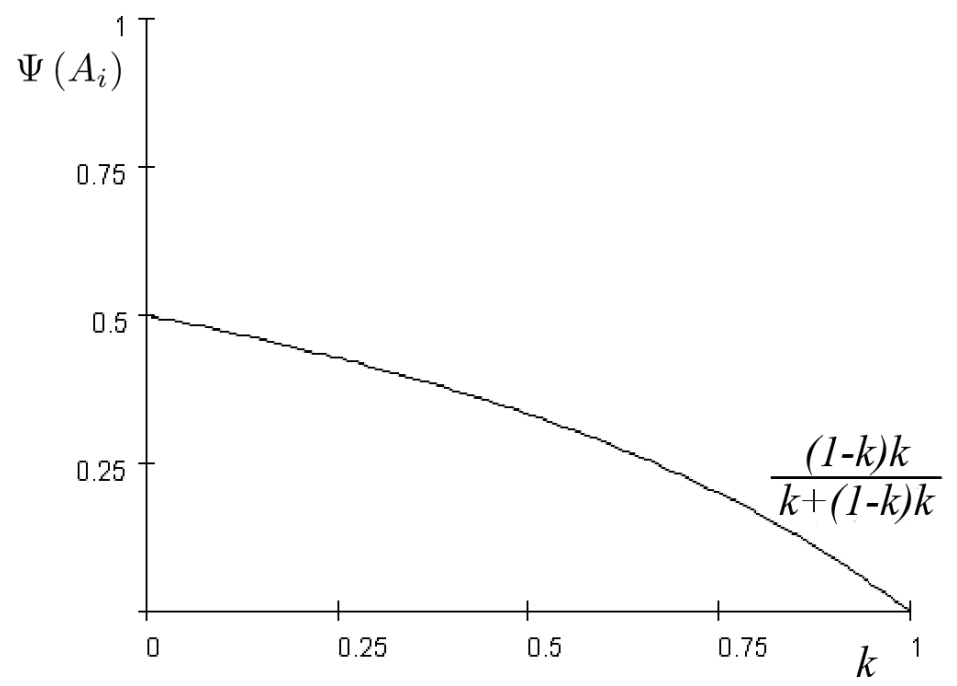

Figure 8: Relative importance of (1-k)k within a LO sell's probability of execution over a range of $k$ 
In general, as the quote drop becomes larger, sellers will be more inclined to opt for $A_{I}$, ceteris paribus. For illustrational purposes, these three possibilities are depicted in Figure 9 with $k=\frac{1}{2}$ and $\Delta=1$. Within the low value segment of $V_{h}-V_{l}$, the quote does not drop but even increases slightly. ${ }^{37}$ Clearly, in this case, LO sellers always opt for $A_{I I}$ as it offers a higher execution probability at a better price. Next, within the intermediate segment, the quote decrease eventually realizes. Still, within this region, the difference between $A_{I}$ and $A_{I I}$ is not large enough to compensate for the lower execution probability at $A_{I}$. Hence, sellers will still tend to stick to quoting $A_{I I}$. However, in the high $V_{h}-V_{l}$ segment the quote difference does become large enough to compensate the lower execution probability, implying sellers will now opt for $A_{I}$. More precisely, sellers could be shown to be indifferent between both quotes when $V_{h}-V_{l}=6.75$ (i.e., where $\frac{3}{4}\left(V_{l}+3-V_{l}\right)=\frac{1}{2}\left(\frac{2}{3} 6.75+V_{l}-V_{l}\right) .{ }^{38}$ Hence, for $k=\frac{1}{2}$, the seller's trade-off between the execution probability decrease and the quote increase is perfectly balanced at $V_{h}-V_{l}=6.75$.

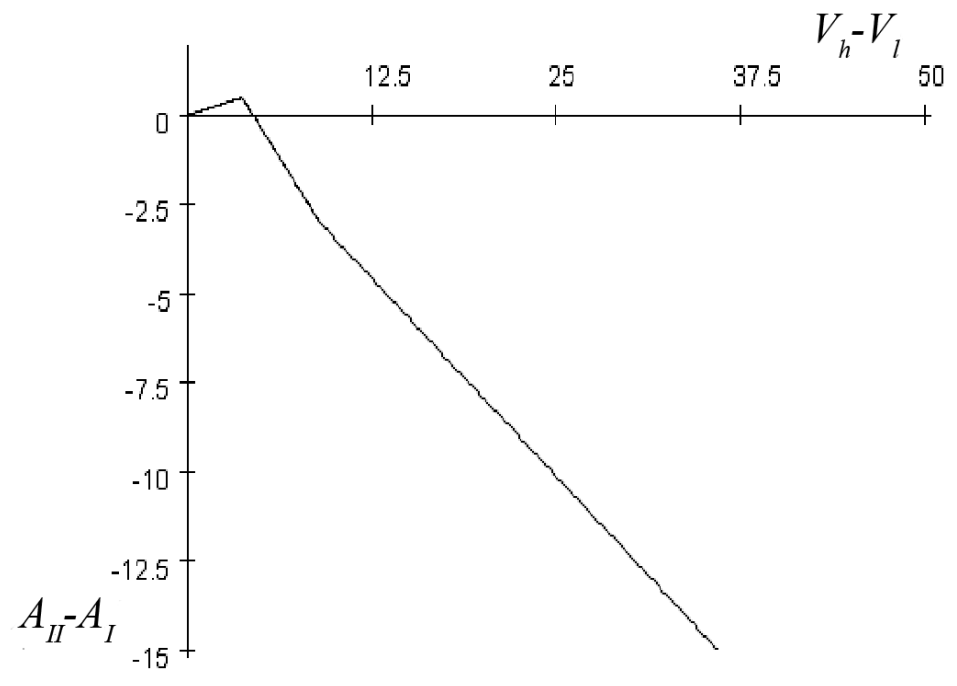

Figure 9: Illustration of the quote decrease with $\mathrm{k}=0.5$ and tick size equal to 1

\footnotetext{
${ }^{37}$ Hence, within this region, the undercutting-proof quote $A_{I I}$ is even positioned higher than $A_{I}$. This is due to the fact that the indifference condition is most binding in Equilibrium $I I$ (i.e. $\left.M=\frac{k}{1-2 k+3 k^{2}-k^{3}}\left(V_{h}-V_{l}\right)+V_{l}\right)$.

${ }^{38}$ Thus, as shown before, in practice, quote $A_{I I}=\frac{2}{9}\left(V_{h}-V_{l}\right)+V_{l}+1$ for $V_{h}-V_{l} \geq 9$ will never realize.
} 
At different values of $k$, the borders between the different $V_{h}-V_{l}$ segments somewhat alter. As before, all depends on the trade-off between quote decrease and execution probability increase:

(i) "low $k$ ": low quote decrease versus high execution probability increase

$$
\text { ( } \Rightarrow \text { tendency for } A_{I I} \text { ); }
$$

(ii) "intermediate $k$ " : high quote decrease versus intermediate execution probability increase

$$
\text { ( } \Rightarrow \text { tendency for } A_{I} \text { ); }
$$

(iii) "high $k$ ": low quote decrease versus low execution probability increase

$$
\text { ( } \Rightarrow \text { tendency for } A_{I I} \text { ). }
$$

For instance, at $k \approx 0$ the execution probability increase is relatively significant, whereas the quote drop is small and even only grows slightly when $V_{h}-V_{l}$ is increased. In this case, sellers only consider shifting from $A_{I I}$ to $A_{I}$ at very high levels of $V_{h}-V_{l}$ at which the quote drop eventually becomes sufficiently large to compensate for the execution probability increase. As an illustration, Figures 10 and 11 depict the quote difference over a range of $V_{h}-V_{l}$ for $k=\frac{1}{10}$ and $k=\frac{9}{10}$ (with $\Delta=1$ ). Do note that, in general, at all values of $k$, the overall pattern remains. Hence, while LO sellers opt for $A_{I I}$ given a low $V_{h}-V_{l}$ value, with high $V_{h}-V_{l}$ values they will post at $A_{I}$. This pattern also clearly realizes in Figure 6. The curve discerning Equilibrium $I$ from Equilibrium $I I$ is skewed to the right due to the relative importance of the execution probability increase at low values of $k$. 


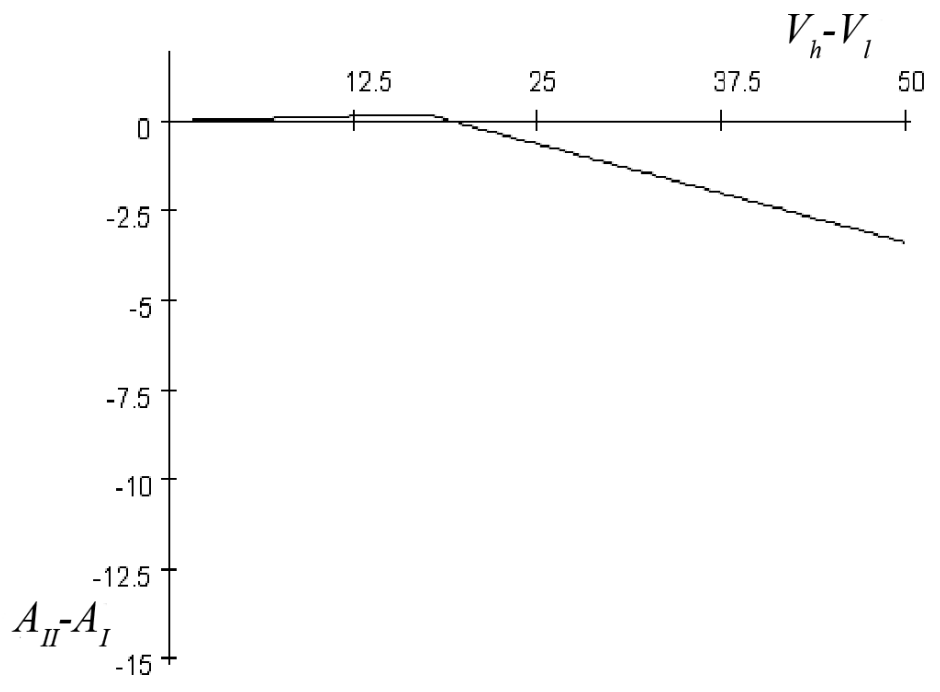

Figure 10: Illustration of the quote decrease with $\mathrm{k}=0.1$ and tick size equal to 1

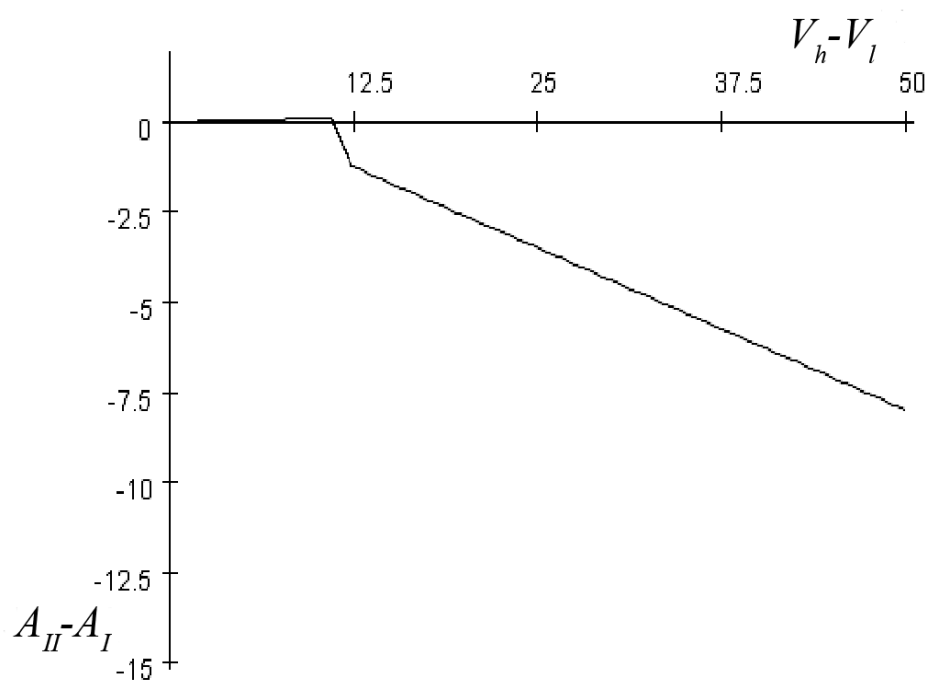

Figure 11: Illustration of the quote decrease with $\mathrm{k}=0.9$ and tick size equal to 1 


\subsubsection{Model parametrization}

To further analyze traders' trading decisions and the resulting limit order book state in a concrete setting, a simple version of the model (i.e., with $k=1 / 2$ and $\Delta=1)$ will now be derived. At these parameter values $M=\min \left[\frac{4}{5}\left(V_{h}-V_{l}\right) ; \max \left(3 ; \frac{2}{9}\left(V_{h}-V_{l}\right)+1\right)\right]+$ $V_{l}$ and traders' quoting behavior occurs as follows:

(i) for $V_{h}-V_{l}<3.75$ (where $\left.M=\frac{4}{5}\left(V_{h}-V_{l}\right)+V_{l}\right)$ :

within this $V_{h}-V_{l}$-region, we are in Equilibrium $I I$, all buyers quote:

$$
B_{I I}^{*}=\frac{3}{5}\left(V_{h}-V_{l}\right)+V_{l},
$$

and all sellers quote:

$$
A_{I I}^{*}=\frac{4}{5}\left(V_{h}-V_{l}\right)+V_{l},
$$

implying a spread of:

$$
S^{*}=\frac{1}{5}\left(V_{h}-V_{l}\right) \text {. }
$$

(ii) for $3.75<V_{h}-V_{l}<6.75$ (where $M=V_{l}+3$ ):

within this $V_{h}-V_{l}$-region, we are in Equilibrium $I I$, all buyers quote:

$$
B_{I I}^{*}=V_{l}+\frac{9}{4}
$$

and all sellers quote:

$$
A_{I I}^{*}=V_{l}+3,
$$

implying a spread of:

$$
S^{*}=\frac{3}{4} \text {. }
$$

(iii) for $6.75<V_{h}-V_{l}<9$ (where $M=V_{l}+3$ ):

within this $V_{h}-V_{l}$-region, we are in Equilibrium $I-N R$, all buyers quote:

$$
B_{I}^{*}=\frac{1}{3}\left(V_{h}-V_{l}\right)+V_{l},
$$

and all first-in-line sellers quote:

$$
A_{I}^{*}=\frac{2}{3}\left(V_{h}-V_{l}\right)+V_{l},
$$

implying a spread of:

$$
S^{*}=\frac{1}{3}\left(V_{h}-V_{l}\right) \text {. }
$$

Further, in equilibrium, all sellers quote:

- $A_{i}-1$ when the current best quote is $A_{i}$ lying within the range $\left(V_{l}+\frac{11}{2}, \frac{2}{3}\left(V_{h}-V_{l}\right)+V_{l}\right)$,

- $V_{l}+3$ when the current best quote is lying within the range $\left[V_{l}+3, V_{l}+\frac{11}{2}\right]$,

- $V_{l}+3$ when the current best quote is $V_{l}+3$,

with $\tilde{A}=V_{l}+\frac{9}{2}$ and $A_{I I}=V_{l}+3$. 
(iv) for $V_{h}-V_{l}>9$ (where $\left.M=\frac{2}{9}\left(V_{h}-V_{l}\right)+V_{l}+1\right)$ :

within this $V_{h}-V_{l}$-region, we are in Equilibrium $I-R$, all buyers quote:

$$
B_{I}^{*}=\frac{1}{3}\left(V_{h}-V_{l}\right)+V_{l},
$$

and all first-in-line sellers quote:

$$
A_{I}^{*}=\frac{2}{3} \cdot\left(V_{h}-V_{l}\right)+V_{l},
$$

implying a spread of:

$$
S^{*}=\frac{1}{3}\left(V_{h}-V_{l}\right) .
$$

Further, in equilibrium, all sellers quote:

- $A_{i}-1$ when the current best quote is $A_{i}$ lying within the range $\left(\frac{1}{3}\left(V_{h}-V_{l}\right)+\frac{5}{2}+V_{l}, \frac{2}{3}\left(V_{h}-V_{l}\right)+V_{l}\right)$,

- $\frac{2}{9}\left(V_{h}-V_{l}\right)+V_{l}+1$ when the current best quote is lying within the range $\left[\frac{2}{9}\left(V_{h}-V_{l}\right)+1+V_{l}, \frac{1}{3}\left(V_{h}-V_{l}\right)+\frac{5}{2}+V_{l}\right]$,

- $\frac{2}{3}\left(V_{h}-V_{l}\right)+V_{l}$ when the current best quote is $\frac{2}{9}\left(V_{h}-V_{l}\right)+1+V_{l}$,

with $\tilde{A}=\frac{1}{3}\left(V_{h}-V_{l}\right)+\frac{3}{2}+V_{l}$ and $A_{I I}=\frac{2}{9}\left(V_{h}-V_{l}\right)+V_{l}+1$.

Normalizing $V_{l}$ to zero for illustrational purposes (i.e., $V_{l}$ merely acts as $y$ intercept), Figure 12 depicts quote submission behavior by buyers and sellers. The slim lines represent the quotes posted by buyers and first-in-line sellers, whereas the bold segment of the ask quote line represents the final ask quote that is reached in the undercutting process which is the result of direct competition among sellers. ${ }^{39}$ Further, Figure 13 depicts the initial spread for both equilibria.

We find that for values of $V_{h}-V_{l}$ lower than 3.75 , the spread is very tight and increases slightly. Next, for values of $V_{h}-V_{l}$ between 3.75 and 6.75 , it remains constant. Finally, at $V_{h}-V_{l}$ values exceeding 6.75, i.e. where Equilibrium $I$ replaces Equilibrium $I I$, it again starts to increase this time at a higher rate than before. Clearly, given the equilibrium structure, this spread will be undercut by consecutively arriving sellers, which is accounted for by the current seller in her execution probability. Note that the evolution of the quote and spread curves at other values of $k$ is quite similar. However, the length of the quote intervals and the associated quote level will be different. Moreover, the spread will become tighter. Figures 14, 15, 16 and 17 depict spread and quote levels for $k=0.1$ and $k=0.9$, respectively. Note that throughout these graphs, again $V_{l}$ is normalized to zero for illustrational purposes.

\footnotetext{
${ }^{39}$ Note that this initial process may ultimately pull the ask quote lower than the bid quote, resulting in a negative virtual spread. An illustration of an undercutting process in Equilibrium $I-R$ is provided later within this subsection.
} 


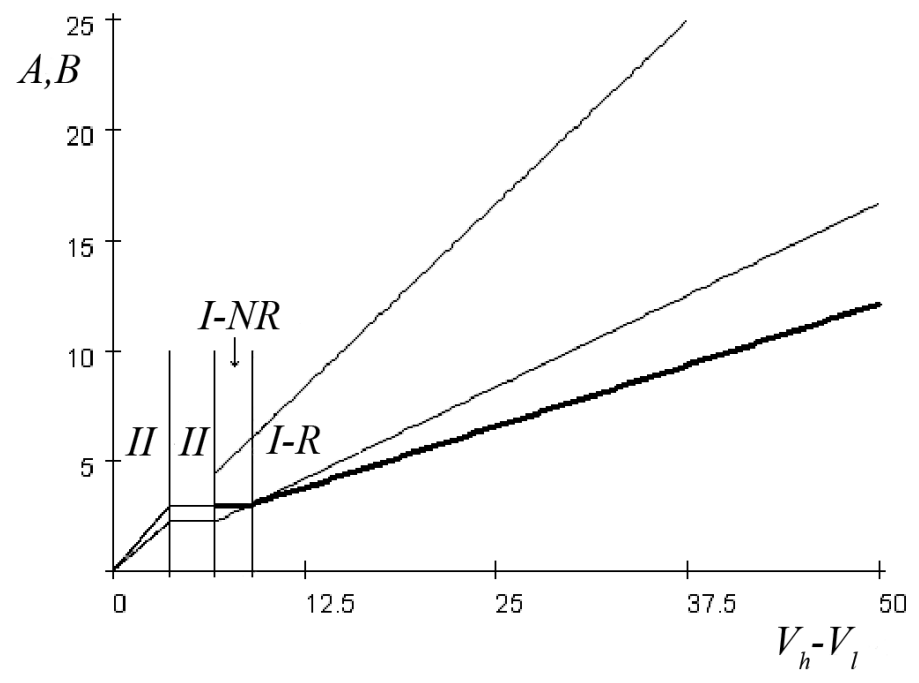

Figure 12: Bid and ask quotes with $\mathrm{k}=0.5$ and tick size equal to 1

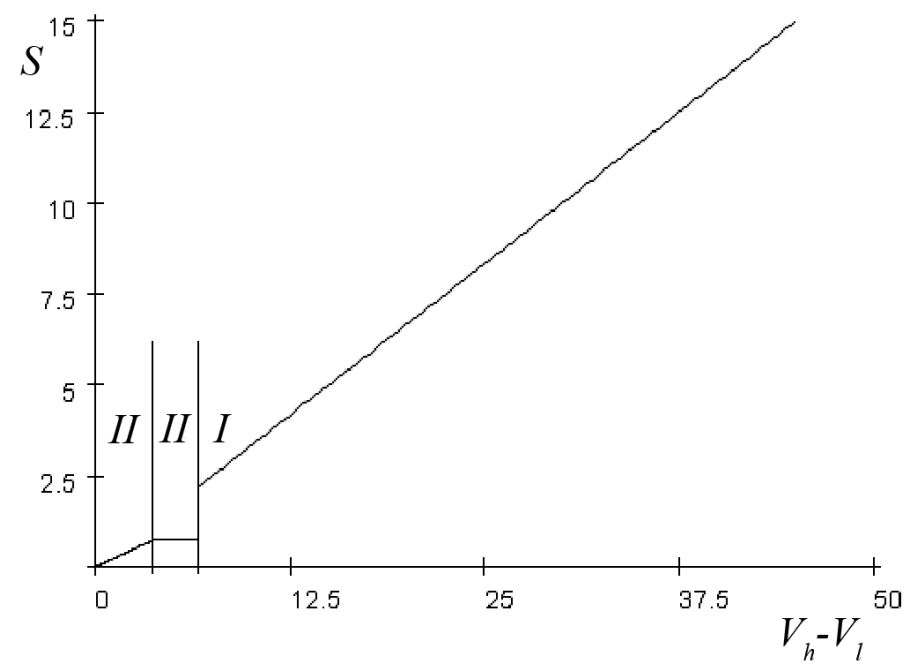

Figure 13: Initial virtual spread with $\mathrm{k}=0.5$ and tick size equal to 1 


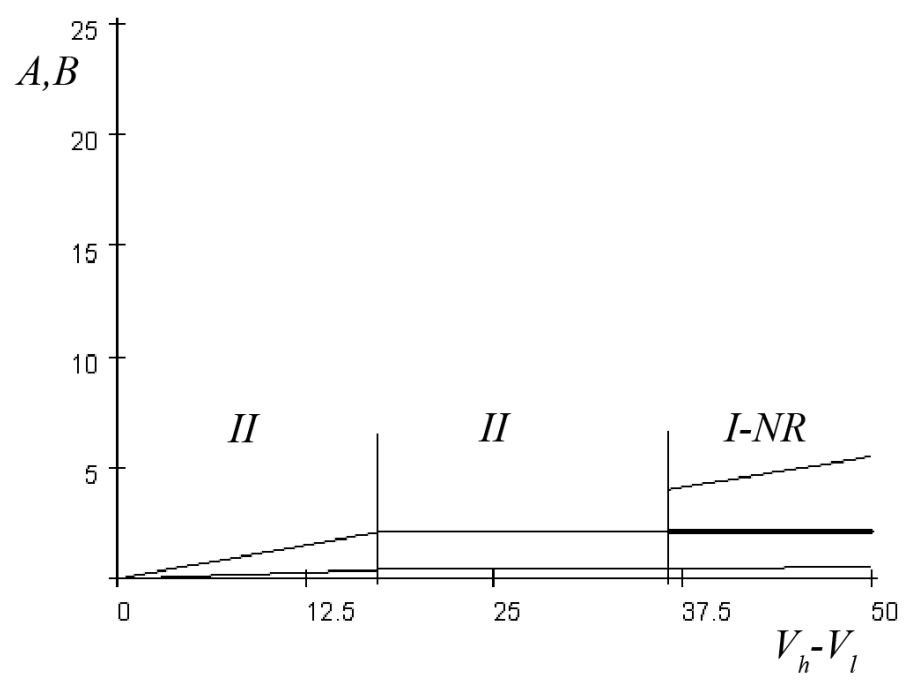

Figure 14: Bid and ask quotes with $\mathrm{k}=\mathbf{0 . 1}$ and tick size equal to 1

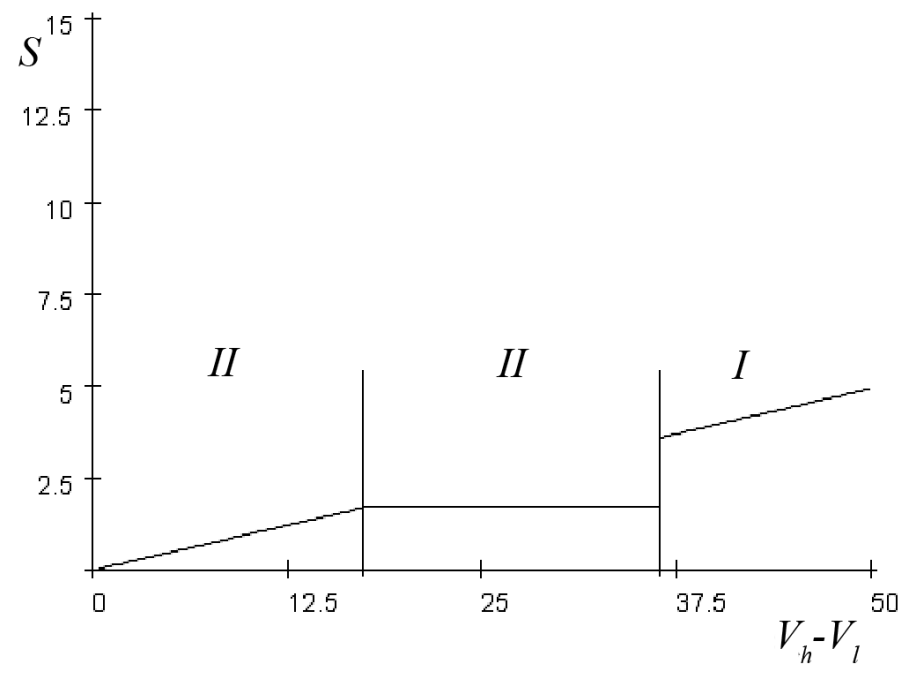

Figure 15: Initial virtual spread with $\mathrm{k}=0.1$ and tick size equal to 1 


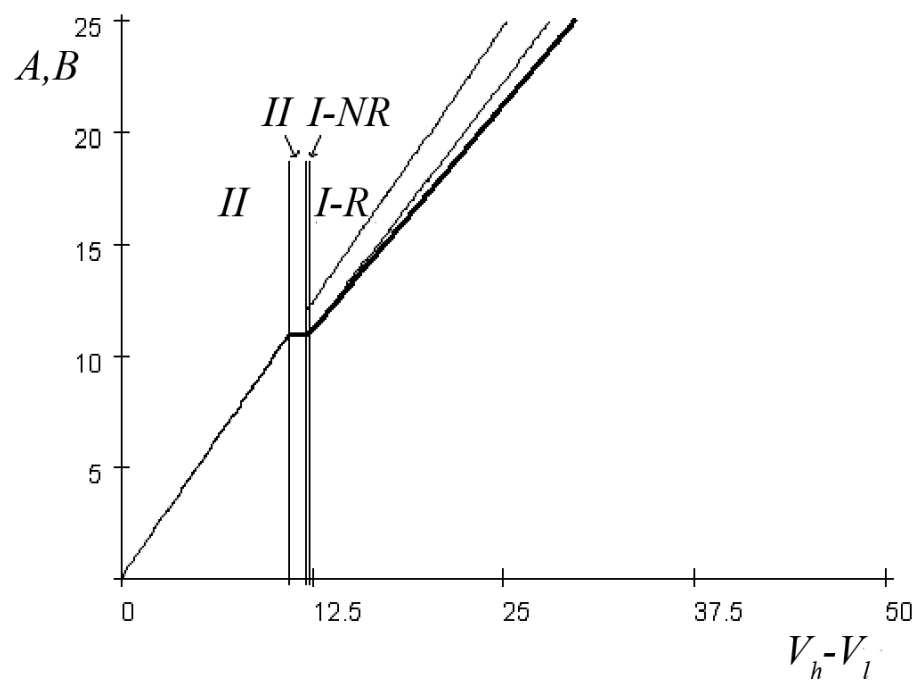

Figure 16: Bid and ask quotes with $\mathrm{k}=0.9$ and tick size equal to 1

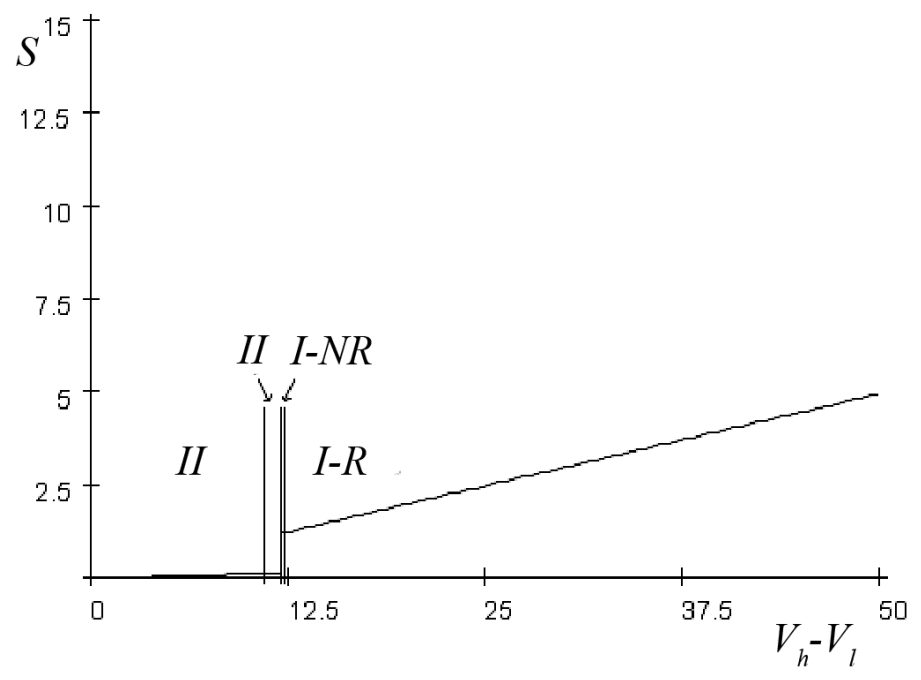

Figure 17: Initial virtual spread with $\mathrm{k}=\mathbf{0 . 9}$ and tick size equal to 1 
As an illustration of the undercutting process in Equilibrium $I-R$, consider the following parameter values: $V_{l}=3, V_{h}=21, \Delta=1, k=1 / 2$ and $\Delta=1$. As $V_{h}-V_{l}=18>9$, indeed we are located in Equilibrium $I-R$ for $k=\frac{1}{2}$. Hence, all buyers quote $B_{I}^{*}=9$, and all first-in-line sellers quote $A_{I}^{*}=15$, which implies a spread of $S_{I}^{*}=6$. Further, in equilibrium, all sellers quote (i) $A_{i}-1$ when the current best quote is $A_{i}$ lying within the price range $(11.5,15)$, (ii) 8 when the current best quote is lying within the range $[8,11.5]$, and (iii) 15 when the current best quote is 8 , with $\tilde{A}=10.5$ and $A_{I I}=8$. The equilibrium path for $A_{I}$ is illustrated in Figure 18. Starting from an initial ask equal to 15 , the consecutive arrival of sellers first leads to tick-by-tick undercutting until $A=11$. Consequently, the quote difference between $A_{I}$ and $A_{I I}$ is skimmed off step by step, implying quoting $A_{I I}=8$ becomes relatively more attractive. At $A=11$, undercutting by a single tick is no longer the most profitable strategy for the next seller arriving. Instead, she prefers to immediately drop the ask quote to its undercutting-proof level $A_{I I}=8$. The most profitable strategy for the incoming seller at that point is to revert back to the initial quote, at which the undercutting process starts over if consecutive sellers keep arriving.

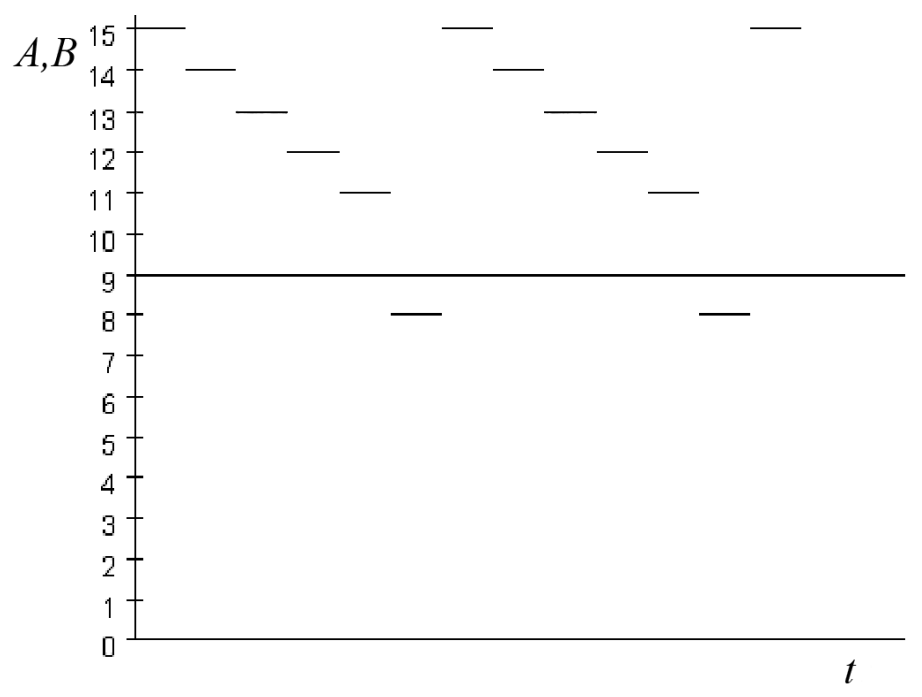

Figure 18: Illustration of equilibrium path ask quote within Equilibrium I-R 


\subsubsection{Expected trading gains}

In the remainder of this subsection, I will investigate how total trading gains are expected to be distributed between both parties trading throughout the different equilibria. ${ }^{40}$ This analysis will provide insights on the issue whether or not patient traders are able to exploit their ability to wait in a limit order market setting and extract trading gains from their impatient counterparties. The results presented will be derived from the simplified version of the model (i.e., with $k=1 / 2$ and $\Delta=1$ ) presented above. Note, however, that the patterns produced at more extreme values of $k$ are very similar. Further, I will only consider expected trading gains for first-in-line sellers. These gains present an upper bound to those of consecutively arriving sellers. Consequently, the provided expected trading gains represent a lower bound for buyers.

Figure 19 depicts the distribution of the expected gains from trading between the two parties involved as a proportion of the total trading gains that are to be distributed. As a benchmark, the slim horizontal lines represent how total trading gains are expected to be distributed in the setting where both buyers' and sellers' trading horizon is restricted to a single period (i.e., as in Foucault (1995, 1999) and Handa, Schwartz and Tiwari (2003)). The bottom slim horizontal line delineates sellers' expected trading gains, whereas the middle one depicts the addition of the expected buyers' trading gains. In expected terms, both trading parties receive a symmetric and constant partition of total trading gains of $\frac{1}{3}$. A residual of $\frac{1}{3}$ is lost due to non-execution of some submitted limit orders. In turn, the bottom and top bold lines represent sellers' and buyers' expected partition of the total trading gains, respectively. Apparently, for total trading gains values lower than 3.75 (i.e., within Equilibrium $I I$ with $\left.M=\frac{4}{5}\left(V_{h}-V_{l}\right)+V_{l}\right)$, in expected terms sellers receive $\frac{3}{5}$ of total trading gains, whereas buyers only earn $\frac{1}{5}$. Thus, within this $V_{h}-V_{l}$-region sellers clearly manage to exploit their patience advantage, as they gain $\frac{4}{15}$. They are able to do so as the indifference condition is the most binding one within this region. As such, their limit order quote only reflects the need to make incoming buyers just willing to submit a market order. They do not have to deal with direct competition from a potentially incoming seller, implying their limit order's exposure is automatically two periods. The sellers appear to gain partly at the expense of the buyers (which lose $\frac{2}{15}$ ), but also due to an equivalent reduction of the non-execution residual. Thus, a higher percentage of total trading gains is expected to be distributed over both parties within this setting as compared to the benchmark, but the distribution occurs in an asymmetric way. Moving on to the region in which $3.75<V_{h}-V_{l}<6.75$ (i.e., within Equilibrium $I I$ with $M=V_{l}+3$ ), we observe direct competition between sellers kicks in, obliging them to submit their respective orders at an undercutting-proof quote which now truly reflects the threat of direct competition. Resultingly, we observe sellers' (buyers') expected trading gains gradually reducing (increasing) towards the benchmark value of $\frac{1}{3}$. Moreover, as mentioned in Subsections 4.1 and 4.2, due to this direct com-

\footnotetext{
${ }^{40}$ Do note that as the expected ex ante trading gains build on rational trader behavior, they are identical to the "average" realized ex post gains.
} 
petition a unique divergence arises between the buyers' expected trading gains from submitting a limit order (bold solid line) and from submitting a market order (dashed line). In case the option arises (i.e., a seller's limit order at $A_{I I}$ is available), submitting a market order boosts the buyer's expected trading gains, the severe direct competition between sellers brings her a bonus. Finally, within the region in which $V_{h}-V_{l}>6.75$ (i.e., within Equilibrium $I$ ), direct competition (and the resulting undercutting process) keeps sellers' expected trading profits down to their benchmark values. ${ }^{41}$ As such, the design of the limit order market prevents sellers from exploiting their patience advantage. Moreover, if they do not arrive first-in-line, due to undercutting they even lose with respect to the benchmark while directly transferring their trading gains to the buyers.

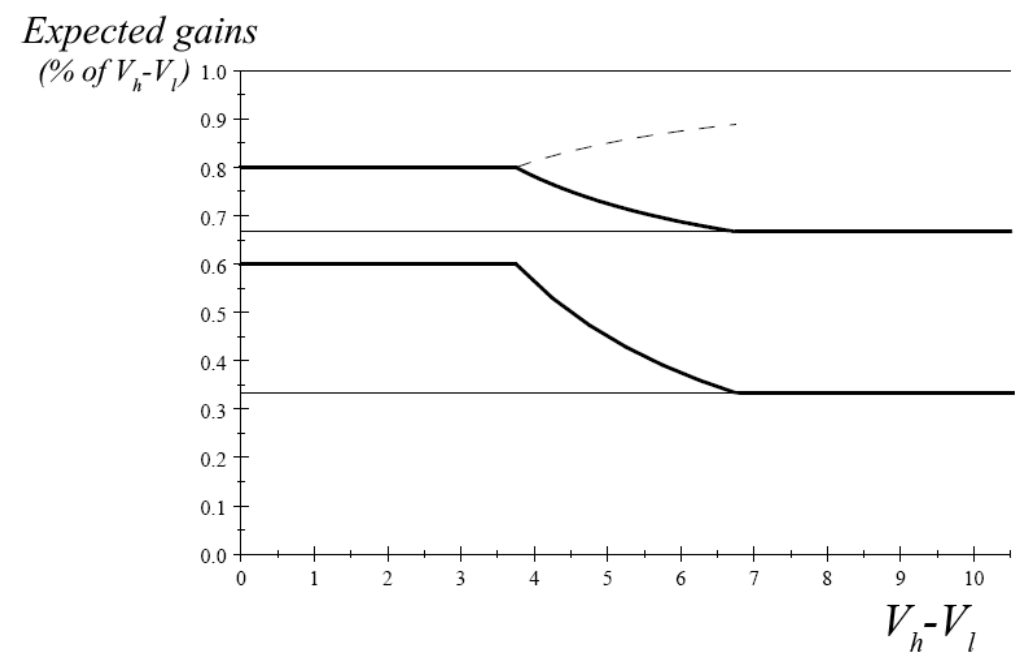

Figure 19: Expected trading profits for first-in-line sellers and buyers with $\mathrm{k}=0.5$ and tick size equal to 1

\section{Conclusion}

This paper considered a stylized trading game in which sequentially arriving liquidity traders could either opt for a market order, or for a limit order. As their impatience was implicitly linked to their trading orientation, one class of traders was argued to have an extended trading horizon: i.e., sellers were considered to have a trading horizon of two periods, whereas buyers only had a single-period trading scope. ${ }^{42}$ Clearly, as the life span of their submitted limit orders was longer, sellers were granted a natural advantage in supplying liquidity within

\footnotetext{
${ }^{41}$ Note there is no difference in expected trading gains between Equilibrium $I-N R$ and Equilibrium $I-R$.

${ }^{42}$ The case where only buyers have an extended trading horizon is completely symmetric.
} 
this setting. This benefit was hampered, however, by the direct competition arising between consecutively arriving sellers.

Closed-form characterizations for the order submission strategies were obtained when solving for the equilibrium of this dynamic game. These allowed to examine how these forces affect traders' order placement decisions and also yielded insight into the dynamic process of price formation and into the market clearing process of a non-intermediated, order driven market. More specifically, the divergence in how a first-in-line LO seller deals with future direct competition facing different market conditions allowed to derive two stationary equilibria for the delineated trading process. In a first equilibrium, she submits a high ask quote at which an incoming buyer is (just) willing to submit a market order. However, due to direct competition this quote will certainly be undercut should the next trader arriving also be a seller. Hence, the consecutive arrival of a series of sellers could induce an undercutting process of this initial quote exhibiting two potential paths: one with and one without reversion to the initial ask quote. In a second equilibrium, a first-in-line LO seller chooses to protect herself from this undercutting process by quoting a low ask quote which is also "undercutting-proof". As such, she is ensured of a two-period order exposure at the cost of a lower execution price. In both equilibria, an LO buyer is shown to merely adapt to the seller's strategy and set the lowest bid quote at which incoming sellers are willing to submit a MO. Hence, both buyers and sellers account for each other's actions in determining their optimal quote. Note that, in fact, Equilibrium $I$ corresponded to a very stable market in which the same quotes are posted over and over again, whereas Equilibrium $I I$ corresponded to a relatively volatile market (at least regarding the ask quote) in which series of arriving sellers cause the ask quote to drop.

The model's results indicated that the quote choice depends on the total trading gains level (i.e., the difference between buyers' and sellers' private valuations). With low and intermediate total trading gains, arriving sellers tend to opt for the undercutting-proof quote (yielding a two-period order exposure). The higher total trading gains become, however, the more sellers are inclined to quote the high ask quote, as the opportunity cost of sticking to the low undercutting-proof quote becomes too high. This effect became less pronounced when sellers outnumber buyers by far in the market, i.e. when higher direct and indirect competition between sellers arises. In this case, the difference between the high and the low quote was shown to be relatively small. Moreover, the execution risk increase from switching from the high to the low quote was argued to be substantial. Consequently, LO sellers will be eager to expose their order for two periods and hence quote the undercutting-proof quote, even for relatively elevated total trading gains levels. Further, patient traders were shown only to be able to extract trading gains from impatient ones when total trading gains are at a low or intermediate level. With high total trading gains, direct competition among patient traders completely wipes out the advantage of being patient, rendering an outcome equivalent to that of a setting in which both trader types have a single-period horizon. 
Future research will focus on relaxing the 1:2-trading horizon assumption. It would be interesting to investigate what would happen if the divergence in trading horizons would be larger or if both one- and two-period horizon traders would exist (on both sides of the market). In the latter case, direct competition between traders potentially arises on both market sides. Given they have a twoperiod horizon, both buyers and sellers could then submit an order at a quote valid for one or two periods (i.e., choose to run a high or a low execution risk, respectively). When submitting an order for one period (thus only intending to make the arriving counterparty trader in the subsequent period indifferent), a trader should further account for the fact that the counterparty is now extracted from a heterogeneous population. This implies the execution probability of her order hinges upon the particular group of traders she wishes to address. When submitting an order intended to last two periods (thus also accounting for a trader potentially arriving on the same market side in the subsequent period), the extent to which this order is undercutting proof to a trader arrival on the same market side in the subsequent period could now also be made contingent on this arriving trader's type. Clearly, in general, the number of possible strategies for each trader category increases within this setting. As such, the game is strongly complexified. Furthermore, I plan to analyze the impact of introducing uncertainty in the traders' individual valuations and of a change in the tick size on the model's results. 


\section{Appendix: Proofs}

\section{Proof of Proposition 1.}

Assume that the buyer who arrives at time $t$, quotes a bid $B(t)$ if she chooses to post a limit order. With that bidding strategy, she gets an expected utility denoted by $E(U(B(t))$. She will decide to submit a market order if the utility she can obtain by trading immediately at the best ask is greater than the expected utility she can get with a limit order:

$$
V_{h}-A^{m}(t) \geq E\left(U(B(t)) \Rightarrow A^{m}(t) \leq V_{h}-E(U(B(t)) .\right.
$$

Let $B_{0}(t)=V_{h}-E\left(U(B(t))\right.$, the last inequality is equivalent to: $A^{m}(t) \leq$ $B_{0}(t)$. Then the buyer submits a market order if this inequality is satisfied. Otherwise she posts a limit order with a price equal to $B(t)$. The case for a seller is completely symmetric.

$$
\text { Q.e.d. }
$$

\section{Proof of Proposition 2.}

In equilibrium, first-in-line sellers choose to run a high execution risk. As a consequence, the following inequality must be satisfied: $k\left(A_{I}-V_{l}\right)>(k+(1-$ $k) k)\left(A_{I I}-V_{l}\right)$.

This condition insures that they quote an ask equal to $A_{I}$, which is the buyer's cutoff price for doing a MO (i.e., $B_{0}^{*}\left(B_{I}\right)$ ). Buyers will always opt for the lowest bid (i.e., $B_{I}$ ) at which the incoming seller (quoting $A_{I}$ as it always is a first-in-line seller) is indifferent between a $\mathrm{MO}$ and a $\mathrm{LO}$ (i.e., where $B_{I}^{*}=$ $\left.A_{0}^{*}\left(A_{I}\right)\right)$. Therefore the optimal bid and ask are $B_{I}^{*}=A_{0}^{*}\left(A_{I}^{*}\right)$ and $A_{I}^{*}=B_{0}^{*}\left(B_{I}^{*}\right)$.

According to the definition of the equilibrium, $A_{0}^{*}\left(A_{I}^{*}\right)$ and $B_{0}^{*}\left(B_{I}^{*}\right)$ must satisfy the following equations:

$$
\begin{aligned}
& A_{0}^{*}=V_{l}+E\left(U\left(A_{I}^{*}\right)\right), \\
& B_{0}^{*}=V_{h}-E\left(U\left(B_{I}^{*}\right)\right), \\
& \quad \text { or } \\
& A_{0}^{*}=V_{l}+k\left(A_{I}^{*}-V_{l}\right)=B_{I}^{*}, \\
& B_{0}^{*}=V_{h}-(1-k)\left(V_{h}-B_{I}^{*}\right)=A_{I}^{*} .
\end{aligned}
$$

Solving this system of equations, one could derive closed form solutions for $A_{I}^{*}$ and $B_{I}^{*}$ : 


$$
\begin{aligned}
& B_{I}^{*}=\lambda V_{l}+(1-\lambda) V_{h} \text { with } \lambda=\frac{1-k}{1-(1-k) k}, \\
& A_{I}^{*}=\mu V_{h}+(1-\mu) V_{l} \text { with } \mu=\frac{k}{1-(1-k) k} .
\end{aligned}
$$

Replacing $A_{I}$ and $A_{I I}$ (see proof of Proposition 6) in the initial existence condition inequality stated above in this proof allows us to derive the set of parameters for which this equilibrium exists:

$$
\mu\left(V_{h}-V_{l}\right)-(2-k)\left(M-V_{l}\right)>0 .
$$

$$
\text { Q.e.d. }
$$

\section{Proof of Proposition 3.}

I.1) As we are in Equilibrium $I$, given an empty LOB a first-in-line seller will always submit a $\mathrm{LO}$ at $A_{I}$ (see proof of Proposition $2, k\left(A_{I}-V_{l}\right)>(k+$ $\left.(1-k) k)\left(A_{I I}-V_{l}\right)\right)$.

I.2) A seller arriving in the subsequent period has three options: (i) she joins the queue at $A_{I}$, (ii) she undercuts $A_{I}$ by one tick or (iii) she submits a LO at $A_{I I}$. All other options could easily be proven to be suboptimal as they offer a lower expected payoff. Option (i) renders a payoff of $k^{2}\left(A_{I}-V_{l}\right)$ as undercutting in the next period is anticipated within this equilibrium (see below). Option (ii) renders $k\left(A_{I}-\Delta-V_{l}\right)$ as a payoff. Finally, option (iii) offers $(k+(1-k) k)\left(A_{I I}-V_{l}\right)$ and has a higher execution probability as this is the undercutting-proof quote (i.e., Equilibrium $I I)$. Now, if $k^{2}\left(A_{I}-V_{l}\right)<$ $k\left(A_{I}-\Delta-V_{l}\right)$ is satisfied, the incoming seller will have an incentive to deviate from the queue-joining strategy and undercut $A_{I}$ by one tick, hence opting for option (ii) instead of option (i). This undercutting process potentially continues: if $k^{2}\left(A_{I}-\Delta-V_{l}\right)<k\left(A_{I}-2 \Delta-V_{l}\right)$, in turn the subsequently arriving seller will also have an incentive to deviate from the queue-joining strategy and undercut $A_{I}-\Delta$ further by one tick. Clearly, given the arrival of consecutive sellers in the subsequent periods, this process of undercutting will continue tick by tick as long as traders have an incentive to deviate, i.e. as long as $k^{2}\left(A_{i}-V_{l}\right)<$ $k\left(A_{i}-\Delta-V_{l}\right)$. We will assume that throughout the quote zone described by $I .2$ this relationship always holds. Moreover, for arriving sellers, submitting a LO at the newly chosen quote $A_{i}-\Delta$ should also be more profitable than returning to $A_{I}$ or than immediately shifting down to $A_{I I}$. Accounting for the fact that her order at $A_{i}-\Delta$ will potentially be undercut (as we are within $I .2$ ), this will be the case when the following inequalities are satisfied: $k^{2}\left(A_{I}-V_{l}\right)<k\left(A_{i}-\Delta-V_{l}\right)$ and $(k+(1-k) k)\left(A_{I I}-V_{l}\right)<k\left(A_{i}-\Delta-V_{l}\right)$ where $A_{i}$ is the initial quote upon arrival.

I.3) The tick-by-tick undercutting process described in $I .2$ continues until a

certain quote $\tilde{A}$ is reached. At this quote we will assume, within this particular Equilibrium $I$ that the condition stated last no longer holds, i.e. now $(k+$ $(1-k) k)\left(A_{I I}-V_{l}\right)>k\left(\tilde{A}-\Delta-V_{l}\right)$, whereas the other two conditions are still 
valid. Hence, undercutting $\tilde{A}$ by one tick is now less profitable than immediately dropping down to $A_{I I}$.

I.4) Finally, given a current quote of $A_{I I}$, an arriving seller will revert to $A_{I}$ if this choice offers her a higher payoff then joining the queue at $A_{I I}$. This will be the case if $k^{2}\left(A_{I}-V_{l}\right)>\left(k^{2}+(1-k) k\right)\left(A_{I I}-V_{l}\right)$.

Summarizing, the following conditions need to be satisfied for Equilibrium $I-R$ to exist:

$$
\begin{aligned}
& \text { I.1) (1) } k\left(A_{I}-V_{l}\right)>(k+(1-k) k)\left(A_{I I}-V_{l}\right) \text {, } \\
& \text { I.2) } \forall A_{i} \in\left\langle\tilde{A}+\Delta, A_{I}\right\rangle \text { : } \\
& \text { (2) } k^{2}\left(A_{i}-V_{l}\right)<k\left(A_{i}-\Delta-V_{l}\right) \\
& \Rightarrow \tilde{A}>V_{l}+\frac{\Delta}{1-k}, \quad\langle 1\rangle \\
& \text { (3) } k^{2}\left(A_{I}-V_{l}\right)<k\left(A_{i}-\Delta-V_{l}\right) \\
& \Rightarrow \tilde{A}>k\left(A_{I}-V_{l}\right)+V_{l}, \quad\langle 2> \\
& \text { (4) }(k+(1-k) k)\left(A_{I I}-V_{l}\right)<k\left(A_{i}-\Delta-V_{l}\right) \\
& \Rightarrow \tilde{A}>(2-k)\left(A_{I I}-V_{l}\right)+V_{l}, \quad\langle 3\rangle
\end{aligned}
$$

I.3) $\left(2^{\prime}\right) k^{2}\left(\tilde{A}-V_{l}\right)<k\left(\tilde{A}-\Delta-V_{l}\right)$,

$\left(3^{\prime}\right) k^{2}\left(A_{I}-V_{l}\right)<k\left(\tilde{A}-\Delta-V_{l}\right)$,

$\left(4^{\prime}\right)(k+(1-k) k)\left(A_{I I}-V_{l}\right)>k\left(\tilde{A}-\Delta-V_{l}\right)$,

I.4) (5) $k^{2}\left(A_{I}-V_{l}\right)>\left(k^{2}+(1-k) k\right)\left(A_{I I}-V_{l}\right)$,

where $\tilde{A}$ can be computed as the maximum of the values at the right hand sides of $\langle 1\rangle,\langle 2\rangle$ and $\langle 3\rangle$, i.e. the highest ask quote at which none of the stated restrictions is broken (according to the equilibrium, at $\tilde{A}-\Delta$ restriction $\langle 3\rangle$ will be broken first $\left(\right.$ see $\left.\left(4^{\prime}\right)\right): \tilde{A}=(2-k)\left(M-V_{l}\right)+V_{l}$. Note that restriction (3) (resp. $\left.\left(3^{\prime}\right)\right)$ in fact fully encompasses restriction (2) (resp. $\left.\left(2^{\prime}\right)\right)$ as $k^{2}\left(A_{I}-V_{l}\right) \geq$ $k^{2}\left(A_{i}-V_{l}\right)$. Therefore, from now on we will drop restriction (2) (resp. (2')).

Using this value and the equilibrium quote values determined in Propositions 2 and 6 , these existence conditions could be restated in exogenous parameters:

(1) $\mu\left(V_{h}-V_{l}\right)-(2-k)\left(M-V_{l}\right)>0$,

$(2-4) \tilde{A}=(2-k)\left(M-V_{l}\right)+V_{l}$,

$\left(3^{\prime}\right) k \mu\left(V_{h}-V_{l}\right)-(2-k)\left(M-V_{l}\right)+\Delta<0$,

(4') $\Delta>0$,

(5) $k \mu\left(V_{h}-V_{l}\right)-\left(M-V_{l}\right)>0$. 


\section{Proof of Proposition 4.}

The proof of the non-reverting version of Equilibrium $I$ proceeds along the lines of that of the reverting version.

I.1) As we are in Equilibrium $I$, given an empty LOB a first-in-line seller will always submit a LO at $A_{I}$ (see proof of Proposition $2, k\left(A_{I}-V_{l}\right)>(k+$ $\left.(1-k) k)\left(A_{I I}-V_{l}\right)\right)$.

I.2) A seller arriving in the subsequent period has three options: (i) she joins the queue at $A_{I}$, (ii) she undercuts $A_{I}$ by one tick or (iii) she submits a LO at $A_{I I}$. All other options could easily be proven to be suboptimal as they offer a lower expected payoff. Option (i) renders a payoff of $k^{2}\left(A_{I}-V_{l}\right)$ as undercutting in the next period is anticipated within this equilibrium (see below). Option (ii) renders $k\left(A_{I}-\Delta-V_{l}\right)$ as a payoff. Finally, option (iii) offers $(k+(1-k) k)\left(A_{I I}-V_{l}\right)$ and has a higher execution probability as this is the undercutting-proof quote (i.e., Equilibrium $I I)$. Now, if $k^{2}\left(A_{I}-V_{l}\right)<$ $k\left(A_{I}-\Delta-V_{l}\right)$ is satisfied, the incoming seller will have an incentive to deviate from the queue-joining strategy and undercut $A_{I}$ by one tick, hence opting for option (ii) instead of option (i). This undercutting process potentially continues: if $k^{2}\left(A_{I}-\Delta-V_{l}\right)<k\left(A_{I}-2 \Delta-V_{l}\right)$, in turn the subsequently arriving seller will also have an incentive to deviate from the queue-joining strategy and undercut $A_{I}-\Delta$ further by one tick. Clearly, given the arrival of consecutive sellers in the subsequent periods, this process of undercutting will continue tick by tick as long as traders have an incentive to deviate, i.e. as long as $k^{2}\left(A_{i}-V_{l}\right)<$ $k\left(A_{i}-\Delta-V_{l}\right)$. We will assume that throughout the quote zone described by $I .2$ this relationship always holds. Moreover, for arriving sellers, submitting a LO at the newly chosen quote $A_{i}-\Delta$ should also be more profitable than returning to $A_{I}$ or than immediately shifting down to $A_{I I}$. Accounting for the fact that her order at $A_{i}-\Delta$ will potentially be undercut (as we are within $I .2$ ), this will be the case when the following inequalities are satisfied: $k^{2}\left(A_{I}-V_{l}\right)<k\left(A_{i}-\Delta-V_{l}\right)$ and $(k+(1-k) k)\left(A_{I I}-V_{l}\right)<k\left(A_{i}-\Delta-V_{l}\right)$ where $A_{i}$ is the initial quote upon arrival.

I.3) The tick-by-tick undercutting process described in $I .2$ continues until a certain quote $\tilde{A}$ is reached. At this quote we will assume, within this particular Equilibrium $I$ that the condition stated last no longer holds, i.e. now $(k+$ $(1-k) k)\left(A_{I I}-V_{l}\right)>k\left(\tilde{A}-\Delta-V_{l}\right)$, whereas the other two conditions are still valid. Hence, undercutting $\tilde{A}$ by one tick is now less profitable than immediately dropping down to $A_{I I}$.

I.4) Finally, given a current quote of $A_{I I}$, an arriving seller will join the queue at $A_{I I}$ if this choice offers her a higher payoff than reverting to $A_{I}$. This will be the case if $k^{2}\left(A_{I}-V_{l}\right)<\left(k^{2}+(1-k) k\right)\left(A_{I I}-V_{l}\right)$. 
Summarizing, the following conditions need to be satisfied for Equilibrium $I-N R$ to exist:

I.1) (1) $k\left(A_{I}-V_{l}\right)>(k+(1-k) k)\left(A_{I I}-V_{l}\right)$,

I.2) $\forall A_{i} \in\left\langle\tilde{A}+\Delta, A_{I}\right\rangle$ :

(2) $k^{2}\left(A_{i}-V_{l}\right)<k\left(A_{i}-\Delta-V_{l}\right)$

$$
\Rightarrow \tilde{A}>V_{l}+\frac{\Delta}{1-k}, \quad\langle 1>
$$

(3) $\forall A_{i} \in\left\langle\tilde{A}+\Delta, A_{I}\right\rangle: k^{2}\left(A_{I}-V_{l}\right)<k\left(A_{i}-\Delta-V_{l}\right)$

$$
\Rightarrow \tilde{A}>k\left(A_{I}-V_{l}\right)+V_{l}, \quad\langle 2>
$$

(4) $\forall A_{i} \in\left\langle\tilde{A}+\Delta, A_{I}\right\rangle:(k+(1-k) k)\left(A_{I I}-V_{l}\right)<k\left(A_{i}-\Delta-V_{l}\right)$

$$
\Rightarrow \tilde{A}>(2-k)\left(A_{I I}-V_{l}\right)+V_{l}, \quad\langle 3\rangle
$$

I.3) $\left(2^{\prime}\right) k^{2}\left(\tilde{A}-V_{l}\right)<k\left(\tilde{A}-\Delta-V_{l}\right)$,

$\left(3^{\prime}\right) k^{2}\left(A_{I}-V_{l}\right)<k\left(\tilde{A}-\Delta-V_{l}\right)$,

$\left(4^{\prime}\right)(k+(1-k) k)\left(A_{I I}-V_{l}\right)>k\left(\tilde{A}-\Delta-V_{l}\right)$,

I.4) (5) $k^{2}\left(A_{I}-V_{l}\right)<\left(k^{2}+(1-k) k\right)\left(A_{I I}-V_{l}\right)$,

where $\tilde{A}$ can be computed as the maximum of the values at the right hand sides of $\langle 1\rangle,\langle 2\rangle$ and $\langle 3\rangle$, i.e. the highest ask quote at which none of the stated restrictions is broken (according to the equilibrium, at $\tilde{A}-\Delta$ restriction $\langle 3\rangle$ will be broken first $\left(\right.$ see $\left.\left(4^{\prime}\right)\right): \tilde{A}=(2-k)\left(M-V_{l}\right)+V_{l}$. Note that restriction (3) (resp. $\left.\left(3^{\prime}\right)\right)$ in fact fully encompasses restriction (2) (resp. $\left.\left(2^{\prime}\right)\right)$ as $k^{2}\left(A_{I}-V_{l}\right) \geq$ $k^{2}\left(A_{i}-V_{l}\right)$. Therefore, from now on we will drop restriction (2) (resp. $\left.\left(2^{\prime}\right)\right)$.

Using this value and the equilibrium quote values determined in Propositions 2 and 6 , these existence conditions could be restated in exogenous parameters:

(1) $\mu\left(V_{h}-V_{l}\right)-(2-k)\left(M-V_{l}\right)>0$,

$(2-4) \quad \tilde{A}=(2-k)\left(M-V_{l}\right)+V_{l}$,

$\left(3^{\prime}\right) k \mu\left(V_{h}-V_{l}\right)-(2-k)\left(M-V_{l}\right)+\Delta<0$,

$\left(4^{\prime}\right) \Delta>0$,

(5) $k \mu\left(V_{h}-V_{l}\right)-\left(M-V_{l}\right)<0$.

$$
\text { Q.e.d. }
$$

\section{Proof of Proposition 5.}

Given that buyers only have a trading horizon of one period, each arriving buyer will "reset" the limit order book state as a seller arriving in the subsequent 
period will always be first-in-line. Hence, in this case we return to the initial state of the equilibrium, which is Equilibrium $I$ as $k\left(A_{I}-V_{l}\right)>(k+(1-$ $k) k)\left(A_{I I}-V_{l}\right)$. Resultingly, buyers will always quote $B_{I}$ and consecutive buyer arrivals will not induce undercutting behavior.

Q.e.d.

\section{Proof of Proposition 6.}

In equilibrium, first-in-line sellers choose to run a low execution risk. As a consequence, the following inequality must be satisfied: $k\left(A_{I}-V_{l}\right)<(k+$ $(1-k) k)\left(A_{I I}-V_{l}\right)$. This condition insures that they quote an ask equal to $A_{I I}$, which is at most equal to the cutoff price of a buyer for doing a MO (i.e., $B_{0}^{*}\left(B_{I I}\right)$, see Lemma 3.1). Buyers will always opt for the lowest bid (i.e., $B_{I I}$ ) at which the incoming seller (quoting $A_{I I}$ as it always is a first-in-line seller) is indifferent between a $\mathrm{MO}$ and a $\mathrm{LO}$ (i.e., $B_{I I}^{*}=A_{0}^{*}\left(A_{I I}\right)$ ). Therefore the optimal bid and ask are $B_{I I}=A_{0}^{*}\left(A_{I I}\right)$ and $A_{I I} \leq B_{0}^{*}\left(B_{I I}\right)$.

According to the definition of the equilibrium, $A_{0}^{*}\left(A_{I I}\right)$ and $B_{0}^{*}\left(B_{I I}\right)$ must satisfy the following equations:

$$
\begin{aligned}
& A_{0}^{*}=V_{l}+E\left(U\left(A^{*}\right)\right), \\
& B_{0}^{*}=V_{h}-E\left(U\left(B^{*}\right)\right) . \\
& \quad \text { or } \\
& A_{0}^{*}=V_{l}+(k+(1-k) k)\left(A_{I I}^{*}-V_{l}\right)=B_{I I}^{*}, \\
& B_{0}^{*}=V_{h}-(1-k)\left(V_{h}-B_{I I}^{*}\right) \geq A_{I I}^{*} .
\end{aligned}
$$

From this system of equations, one could derive boundaries for $A_{I I}^{*}$ and $B_{I I}^{*}$ :

$$
\begin{aligned}
& B_{I I}^{*} \leq \frac{k^{2}(2-k)}{1-2 k+3 k^{2}-k^{3}}\left(V_{h}-V_{l}\right)+V_{l} \\
& \quad \text { and } \\
& A_{I I}^{*} \leq \frac{k}{1-2 k+3 k^{2}-k^{3}}\left(V_{h}-V_{l}\right)+V_{l} \cdot{ }^{43} \quad\langle 4>
\end{aligned}
$$

However, these boundary values do not necessarily represent the equilibrium quotes. For $B_{I I}^{*}$ and $A_{I I}^{*}$ to be the equilibrium quotes, the current seller needs to verify that her quote will not be undercut by the seller arriving in the subsequent period. Otherwise the stated execution probability would be an overestimation of its true value. For $A_{I I}^{*}$ to be undercutting-proof, at least one of the next two conditions needs to be satisfied:

\footnotetext{
${ }^{43}$ Note: always $1-2 k+3 k^{2}-k^{3}>0$.
} 
(i) $\left.(k+(1-k) k)\left(A_{I I}-\Delta-V_{l}\right) \leq k^{2}\left(A_{I}-V_{l}\right)=\frac{k^{3}}{1-(1-k) k}\left(V_{h}-V_{l}\right)\right)$ (i.e., for the seller arriving in the subsequent period undercutting $A_{I I}$ by one tick renders at most the payoff of submitting a LO at $A_{I}$ ),

(ii) $(k+(1-k) k)\left(A_{I I}-\Delta-V_{l}\right) \leq\left(k^{2}+(1-k) k\right)\left(A_{I I}-V_{l}\right)$ (i.e., for the seller arriving in the subsequent period undercutting $A_{I I}$ by one tick renders at most the payoff of joining the queue at $A_{I I}$ ).

These conditions could be rewritten as follows, respectively:

$$
\begin{aligned}
& A_{I I} \leq \frac{k \mu}{(2-k)}\left(V_{h}-V_{l}\right)+\Delta+V_{l}, \quad\langle 5\rangle \\
& A_{I I} \leq \Delta \frac{2-k}{1-k}+V_{l} . \quad\langle 6\rangle
\end{aligned}
$$

So far, we derived three boundary conditions for $A_{I I}$. To determine the equilibrium quote $A_{I I}^{*}$, we take the right hand sides of $\langle 4\rangle,\langle 5\rangle$ and $\langle 6\rangle$ and solve: $A_{I I}^{*}=\min [$ R.H.S. $\langle 4\rangle, \max ($ R.H.S. $\langle 5\rangle$, R.H.S. $\langle 6\rangle)]$ which we define as $M$ for notational convenience. ${ }^{44}$ Consequently, $B_{I I}^{*}$ could be found as: $B_{I I}^{*}=$ $V_{l}+(k+(1-k) k)\left(M-V_{l}\right)$.

Replacing $A_{I}$ and $A_{I I}$ in the initial existence condition inequality stated above in this proof allows us to derive the set of parameters for which this equilibrium exists:

$$
\mu\left(V_{h}-V_{l}\right)-(2-k)\left(M-V_{l}\right)<0 .
$$

$$
\text { Q.e.d. }
$$

\section{Proof of Proposition 7.}

$I I .1)$ As we are in Equilibrium $I I$, given an empty LOB a first-in-line seller will always submit a LO at $A_{I I}$ (see proof of Proposition $6, k\left(A_{I}-V_{l}\right)<$ $\left.(k+(1-k) k)\left(A_{I I}-V_{l}\right)\right)$. A seller arriving in the subsequent period has two options: (i) she joins the queue at $A_{I I}$ or (ii) she submits a LO at $A_{I}$. All other options could easily be proven to be suboptimal as they offer a lower expected payoff. Option (i) renders $\left(k^{2}+(1-k) k\right)\left(A_{I I}-V_{l}\right)$ as a payoff, whereas option (ii) provides $k^{2}\left(A_{I}-V_{l}\right) \cdot{ }^{45}$ Hence, if $k^{2}\left(A_{I}-V_{l}\right)<\left(k^{2}+(1-k) k\right)\left(A_{I I}-V_{l}\right)$, the

\footnotetext{
${ }^{44}$ Note that the use of the max-operator reflects the fact that only one of both restrictions $\langle 5\rangle$ or $\langle 6\rangle$ needs to be satisfied, i.e. the one that is least binding. Which one is least binding depends on the direction of the inequality in the reversion existence condition $k^{2}\left(A_{I}-V_{l}\right) \gtrless$ $\left(k^{2}+(1-k) k\right)\left(A_{I I}-V_{l}\right)$ (i.e., different for Equilibrium $I I-N R$ vs. $\left.I I-R\right)$. The use of the min-operator allows to account for the fact that incoming sellers need to be willing to submit a $\mathrm{MO}$ at the quoted ask.

${ }^{45}$ It provides $k^{2}\left(A_{I}-V_{l}\right)$ as a future arriving seller will always undercut back to $A_{I I}$ due to the following initial equilibrium condition: $k^{2}\left(A_{I}-V_{l}\right) \leq k\left(A_{I}-V_{l}\right)<(k+(1-k) k)\left(A_{I I}-V_{l}\right)$ (cfr. part I.3).
} 
incoming seller will have no incentive to deviate from the queue-joining strategy at $A_{I I}$ and will opt for option (i). Future sellers arriving consecutively will face a similar trading problem, and thus also opt for $A_{I I}$.

Summarizing, the following conditions need to be satisfied for Equilibrium $I I$ to exist in its non-reverting form:

II.1) (1) $k\left(A_{I}-V_{l}\right)<(k+(1-k) k)\left(A_{I I}-V_{l}\right)$,

(2) $k^{2}\left(A_{I}-V_{l}\right)<\left(k^{2}+(1-k) k\right)\left(A_{I I}-V_{l}\right)$.

Implementing the equilibrium quote values determined in Propositions 2 and 6 , these existence conditions could be restated in exogenous parameters:

(1) $\mu\left(V_{h}-V_{l}\right)-(2-k)\left(M-V_{l}\right)<0$,

(2) $k \mu\left(V_{h}-V_{l}\right)-\left(M-V_{l}\right)<0$.

\section{Q.e.d.}

\section{Proof of Proposition 8.}

Given that buyers only have a trading horizon of one period, each arriving buyer will "reset" the limit order book state as a seller arriving in the subsequent period will always be first-in-line. Hence, in this case we return to the initial state of the equilibrium, which is Equilibrium $I I$ as $k\left(A_{I}-V_{l}\right)<(k+(1-$ $k) k)\left(A_{I I}-V_{l}\right)$. Resultingly, buyers will always quote $B_{I I}$ and consecutive buyer arrivals will not induce undercutting behavior.

$$
\text { Q.e.d. }
$$

\section{Proof of Proposition 9.}

The initial spread $S_{I}$ is given by:

$$
\begin{aligned}
& S_{I}=A_{I}^{*}-B_{I}^{*}=\left[\mu V_{h}+(1-\mu) V_{l}\right]-\left[\lambda V_{l}+(1-\lambda) V_{h}\right]=\varphi\left(V_{h}-V_{l}\right) \\
& \quad \text { with } \varphi=k \lambda=(1-k) \mu .
\end{aligned}
$$

The initial spread $S_{I I}$ in case $M=\frac{k}{1-2 k+3 k^{2}-k^{3}}\left(V_{h}-V_{l}\right)+V_{l}$ is given by:

$$
\begin{aligned}
S_{I I} & =A_{I I}^{*}-B_{I I}^{*}=M-\left[V_{l}+(k+(1-k) k)\left(M-V_{l}\right)\right] \\
& =(1-(k+(1-k) k))\left(\frac{k}{1-2 k+3 k^{2}-k^{3}}\left(V_{h}-V_{l}\right)\right) \\
& =\frac{k(1-k)^{2}}{1-2 k+3 k^{2}-k^{3}}\left(V_{h}-V_{l}\right) .
\end{aligned}
$$

The initial spread $S_{I I}$ in case $M=\Delta \frac{2-k}{1-k}+V_{l}$ is given by: 


$$
\begin{aligned}
S_{I I} & =A_{I I}^{*}-B_{I I}^{*}=M-\left[V_{l}+(k+(1-k) k)\left(M-V_{l}\right)\right] \\
& =(1-(k+(1-k) k)) \Delta \frac{2-k}{1-k} \\
& =(1-k)(2-k) \Delta .
\end{aligned}
$$

In the remainder of this proof, we will derive the properties of the functions $S_{I}$ and $S_{I I}$ which are as follows:

(i) $S_{I I}$ if $M=\Delta \frac{2-k}{1-k}+V_{l}$ has a local maximum within the $k \in[0,1]$-interval when $k=0$, where its value is $2 \Delta$. Further, it has a local minimum when $k=1$, where its value is 0 ;

(ii) $S_{I I}$ if $M=\frac{k}{1-2 k+3 k^{2}-k^{3}}\left(V_{h}-V_{l}\right)+V_{l}$ has a local maximum within the $k \in[0,1]$-interval when $k=\frac{1}{3}$, where its value is $\frac{4}{17}\left(V_{h}-V_{l}\right)$. Further, it has a local minimum when $k=0$ and when $k=1$, where its value is 0 ;

(iii) $S_{I}$ is a concave function of $k$, and is maximized when $k=\frac{1}{2}$, where its value is $\frac{1}{3}\left(V_{h}-V_{l}\right)$. Further, it has a local minimum within the $k \in[0,1]$ interval when $k=0$ and when $k=1$, where its value is 0 .

To prove this, first of all, for $S_{I}$ we show that $\varphi$ is maximized at $k=\frac{1}{2}$.

Now:

$\varphi=k \lambda=k \frac{1-k}{1-(1-k) k}$.

Taking the first derivative, we get:

$\frac{\mathrm{d} \varphi}{\mathrm{d} k}=-\frac{2 k-1}{(1-(1-k) k)^{2}}$,

which is equal to 0 when $k=\frac{1}{2}$.

To confirm that $k=\frac{1}{2}$ is indeed the maximum, we take the second derivative with respect to $k$ and get:

$\frac{\mathrm{d}^{2} \varphi}{\mathrm{d} k^{2}}=-\frac{2(2 k-1)^{2}}{(1-(1-k) k)^{3}}-\frac{2}{(1-(1-k) k)^{2}}<0$ when $k=\frac{1}{2}$.

Note that at $k=\frac{1}{2}$ we have $\varphi=\frac{1}{3}$.

As $\varphi$ attains a maximum at $k=\frac{1}{2}$ and has no other extreme value, it must attain minimum value (of 0 ) at the upper and lower bounds of $k$, i.e. at $k=0$ and $k=1$. These findings are confirmed in Figure 20 depicting $S_{I}$. Similar calculations render the local maxima and minima the other spread functions: 
(i) for $S_{I I}$ if $M=\Delta \frac{2-k}{1-k}+V_{l}$ : within the $k \in[0,1]$-interval $k=0$ is the local maximum and $k=1$ is the local minimum, which is illustrated in Figure 21

(ii) for $S_{I I}$ if $M=\frac{k}{1-2 k+3 k^{2}-k^{3}}\left(V_{h}-V_{l}\right)+V_{l}$ : within the $k \in[0,1]$-interval $k=\frac{1}{3}$ is the local maximum and $k=0$ and $k=1$ are the local minima, which is illustrated in Figure 22.

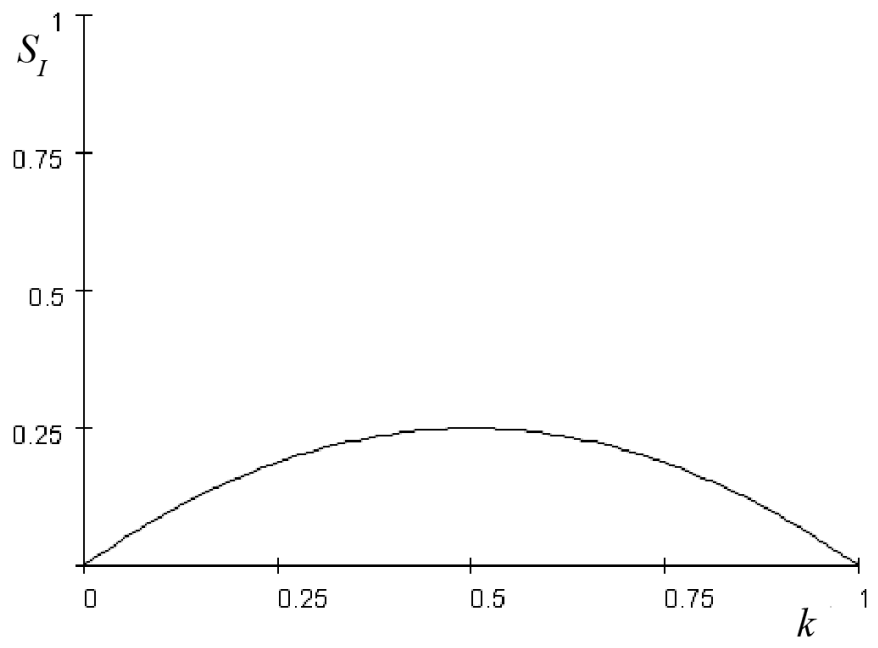

Figure 20: Graphical representation of the spread within Equilibrium I for a range of $\mathrm{k}$ (valid for the high total trading gains segment)

$$
\text { Q.e.d. }
$$




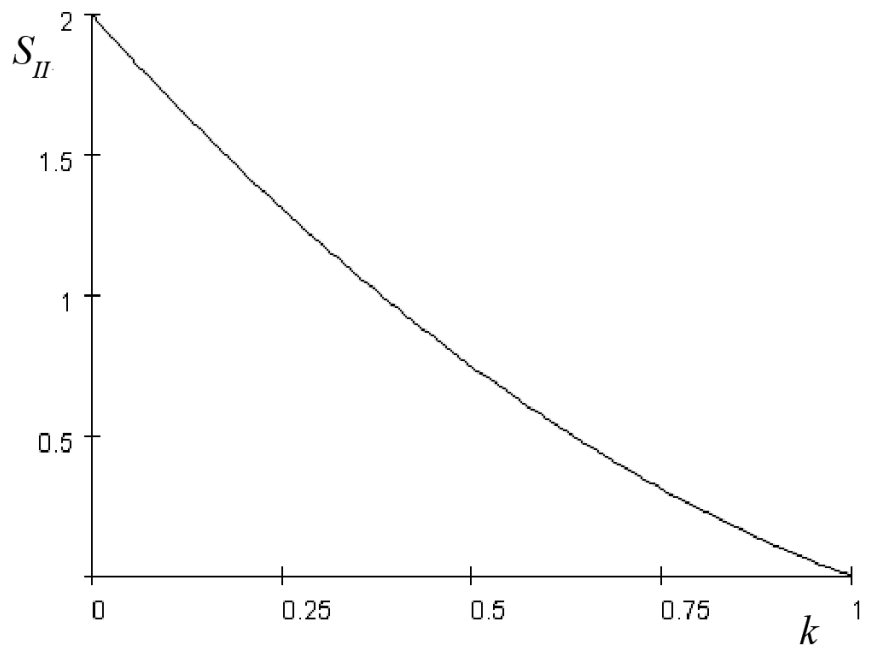

Figure 21: Graphical representation of the spread within Equilibrium II (valid for the intermediate total trading gains segment)

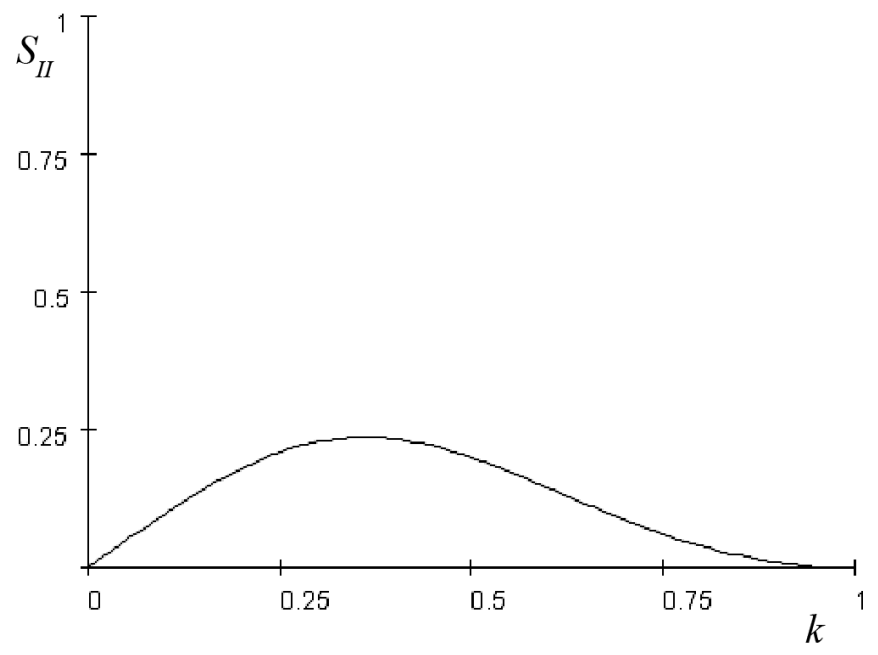

Figure 22: Graphical representation of the spread within Equilibrium II (valid for the low total trading gains segment) 


\section{References}

[1] Angel, J., 1994, Limit versus Market Orders, working paper.

[2] Bernhardt, D. and E. Hughson, 1997, Splitting Orders, Review of Financial Studies 10, pp. 69-101.

[3] Biais, B., D. Martimort and J.-C. Rochet, 2000 Competing Mechanisms in a Common Value Environment, Econometrica 78, pp. 799-837.

[4] Brunnermeier, M.K. and L.H. Pedersen, 2007, Market Liquidity and Funding Liquidity, working paper.

[5] Chacko, G., J. Jurek and E. Stafford, The Price of Immediacy, forthcoming in Journal of Finance.

[6] Chakravarty, S. and C. Holden, 1995, An Integrated Model of Market and Limit Orders, Journal of Financial Intermediation 4, pp. 213-241.

[7] Cordella, T. and T. Foucault, 1999, Minimum Price Variations, Time Priority, and Quote Dynamics, Journal of Financial Intermediation 8, pp. 141-173.

[8] Domowitz, I. and J. Wang, 1994, Auctions as Algorithms: Computerized Trade Execution and Price Discovery, Journal of Economic Dynamics and Control 18(1), pp. 29-60.

[9] Duffie, D., N. Gârleanu and L. Pedersen, 2005, Over-the-counter Markets, Econometrica 73 , pp. 1815-1847.

[10] Edgeworth, F., 1925, The Pure Theory of Monopoly, in Papers Relating to Political Economy, Vol. 1, London: MacMillan, pp. 111-142.

[11] Ewerhart, C. and N. Valla, 2007, Forced Portfolio Liquidation, working paper.

[12] Foucault, T., 1995, Price Formation and Order Placement Strategies in a Dynamic Order Driven Market, working paper.

[13] Foucault, T., 1999, Order Flow Composition and Trading Costs in a Dynamic Limit Order Market, Journal of Financial Markets 2, pp. 99-134.

[14] Foucault, T., O. Kadan and E. Kandel, 2005, Limit Order Book as a Market for Liquidity, Review of Financial Studies 18, pp. 1171-1217.

[15] Glosten, L., 1994, Is the Electronic Open Limit Order Book Inevitable?, Journal of Finance 49, pp. 1127-1161.

[16] Glosten, L. and P. Milgrom, 1985, Bid Ask and Transaction Prices in a Specialist Market with Heterogeneously Informed Traders, Journal of Financial Economics 14, pp. 71-100 
[17] Goettler, R., C. Parlour and U. Rajan, 2005, Equilibrium in a Dynamic Limit Order Market, Journal of Finance 60, pp. 2149-2192.

[18] Handa, P. and R. Schwartz, 1996, Limit Order Trading, Journal of Finance 51, pp. 1835-1861.

[19] Handa, P., R. Schwartz and A. Tiwari, 2003, Quote Setting and Price Formation in an Order Driven Market, Journal of Financial Markets 6, pp.461-489.

[20] Harris, L., 1998, Optimal Dynamic Order Submission Strategies in Some Stylized Trading Problems, Financial Markets, Institutions $\& 3$ Instruments 7, no.2.

[21] Hong, H., J. Scheinkman and W. Xiong, 2006, Asset Float and Speculative Bubbles, Journal of Finance 61, pp. 1073-1117.

[22] Huang, R. and H. Stoll, 1997, The Components of the Bid-Ask Spread: a General Approach, Review of Financial Studies 10, pp. 995-1034.

[23] Jain, P., 2003, Institutional Design and Liquidity on Stock Exchanges, working paper.

[24] Kang, W., A. Hameed and S. Viswanathan, 2006, Stock Market Decline and Liquidity, working paper.

[25] Madhavan, A., 2000, Market Microstructure: A Survey, Journal of Financial Markets 3, pp. 205-258.

[26] Maskin, E. and J. Tirole, 1988, A Theory of Dynamic Oligopoly, II: Price Competition, Kinked Demand Curves, and Edgeworth Cycles, Econometrica 56, pp.571-599.

[27] Parlour, C., 1998, Price Dynamics in Limit Order Markets, Review of Financial Studies 11, pp. 789-816.

[28] Parlour, C. and D. Seppi, 2003, Liquidity-Based Competition for Order Flow, Review of Financial Studies 16, pp. 301-343.

[29] Rock, K., 1996, The Specialist's Order Book and Price Anomalies, working paper.

[30] Rosu, I., 2007, A Dynamic Model of the Limit Order Book, working paper.

[31] Seppi, D., 1997, Liquidity Provision with Limit Orders and a Strategic Specialist, Review of Financial Studies 10, pp. 103-150.

[32] Scheinkman, J. and W. Xiong, 2003, Overconfidence and Speculative Bubbles, Journal of Political Economy 111, pp. 1183-1219.

[33] Shiller, R.J., 2005, Irrational Exuberance, 2nd ed., Princeton University Press. 